\title{
Taming the State-space Explosion in the Makespan Optimization of Flexible Manufacturing Systems
}

\author{
JOÃO BASTOS, JEROEN VOETEN, and SANDER STUIJK, Eindhoven University of Technology,
} The Netherlands

RAMON SCHIFFELERS, Eindhoven University of Technology and ASML, The Netherlands

HENK CORPORAAL, Eindhoven University of Technology, The Netherlands

\begin{abstract}
This article presents a modular automaton-based framework to specify flexible manufacturing systems and to optimize the makespan of product batches. The Batch Makespan Optimization (BMO) problem is NP-Hard and optimization can therefore take prohibitively long, depending on the size of the state-space induced by the specification. To tame the state-space explosion problem, we develop an algebra based on automata equivalence and inclusion relations that consider both behavior and structure. The algebra allows us to systematically relate the languages induced by the automata, their state-space sizes, and their solutions to the BMO problem. Further, we introduce a novel constraint-based approach to systematically prune the statespace based on the the notions of nonpermutation-repulsiveness and permutation-attractiveness. We prove that constraining a nonpermutation-repulsing automaton with a permutation-attracting constraint always reduces the state-space. This approach allows us to (i) compute optimal solutions of the BMO problem when the (additional) constraints are taken into account and (ii) compute bounds for the (original) BMO problem (without using the constraints). We demonstrate the effectiveness of our approach by optimizing an industrial wafer handling controller.
\end{abstract}

CCS Concepts: • Theory of computation $\rightarrow$ Formal languages and automata theory; $\bullet$ Computing methodologies $\rightarrow$ Model verification and validation; $\bullet$ Computer systems organization $\rightarrow$ Embedded and cyber-physical systems;

Additional Key Words and Phrases: Flexible manufactoring systems, formal specification, makespan optimization, max plus linear systems

\section{ACM Reference format:}

João Bastos, Jeroen Voeten, Sander Stuijk, Ramon Schiffelers, and Henk Corporaal. 2021. Taming the Statespace Explosion in the Makespan Optimization of Flexible Manufacturing Systems. ACM Trans. Cyber-Phys. Syst. 5, 2, Article 15 (January 2021), 26 pages.

https://doi.org/10.1145/3426194

\footnotetext{
This research is supported by the Dutch NWO-TTW, carried out as part of the Robust Cyber-Physical Systems (RCPS) program, project number 12694. Research leading to these results has received funding from the EU ECSEL Joint Undertaking under grant agreement no 826452 (project Arrowhead Tools).

The proofs for all Lemmas and Theorems can be found in an Appendix provided with the supplementary material. Authors' addresses: J. Bastos, J. Voeten, S. Stuijk, and H. Corporaal, TUe Department of Electrical Engineering, Eindhoven University of Technology, 5612AP Eindhoven, The Netherlands; emails: joaobastos@me.com, \{j.p.m.voeten, s.stuijk, h.corporaal\}@tue.nl; R. Schiffelers, ASML Veldhoven, The Netherlands; email: ramon.schiffelers@asml.com.

Permission to make digital or hard copies of all or part of this work for personal or classroom use is granted without fee provided that copies are not made or distributed for profit or commercial advantage and that copies bear this notice and the full citation on the first page. Copyrights for components of this work owned by others than ACM must be honored. Abstracting with credit is permitted. To copy otherwise, or republish, to post on servers or to redistribute to lists, requires prior specific permission and/or a fee. Request permissions from permissions@acm.org.

(c) 2021 Association for Computing Machinery.

2378-962X/2021/01-ART15 \$15.00

https://doi.org/10.1145/3426194
}

ACM Transactions on Cyber-Physical Systems, Vol. 5, No. 2, Article 15. Publication date: January 2021. 


\section{INTRODUCTION}

Flexible Manufacturing Systems (FMSs) are Cyber-Physical Systems that perform operations on batches of products. Production and transportation resources (the physical part) carry out operations while a logistics controller (the cyber part) assigns operations to resources and determines their order. Examples of these systems include lithography machines and industrial production printers. During their design, functional requirements (such as keeping the order of products in a batch or avoiding collisions) must be satisfied while at the same time demanding performance requirements must be met, for instance regarding productivity.

In References $[6,19]$ a framework is proposed for the batch-oriented specification and design exploration of FMSs (shown in Figure 1). The novelty of this framework lies in the separation of concerns between specification and optimization, together with its compositional support for requirements specification. The physical system is abstracted into sets of resources, peripherals, and actions. Different pieces of end-to-end deterministic functional behavior are captured as activities that are Directed Acyclic Graphs (DAGs) composed of multiple peripheral actions and dependencies among them. The temporal behavior of each activity is characterized using $(\max ,+)$ algebra. An activity sequence can be constructed to describe more elaborate functional behaviors by considering multiple activities, such as the complete manufacturing of a product where each operation is captured by a single activity. The set of all possible activity sequences is captured as the language accepted by a finite state automaton, called a logistics automaton. Logistics automata can be defined modularly for each product in a batch. The logistics automaton of the batch itself is then obtained by composing these automata by means of a composition operator. Besides logistics requirements a system also exhibits constraints on different product flows, such as resource capacity and safety constraints. These are modularly captured in our framework as constraint automata, and a constraint operator is provided to compose them with logistics automata.

Once the FMS has been specified, the minimal completion time of a batch of products can be computed by solving an optimization problem called the Batch Makespan Optimization (BMO) problem. This BMO problem is NP-Hard [6]. Computing makespan-optimal solutions can therefore take prohibitively long, due to state-space explosion. To keep state-spaces manageable, we complement the work of Reference [6] in this article by developing an algebra of logistics automata based on equivalence and inclusion relations that take both behavioral and structural information into account. The algebra allows us to relate the languages induced by the automata, their state-space sizes, and their solutions to the BMO problem, thereby providing a systematic approach to specify and explore different designs of FMSs. To the best of our knowledge, this is the first work in which an algebraic framework is developed to reason qualitatively about state-space sizes of FSMs. Further, we introduce a novel constraint-based approach to systematically prune the state-space based on the notions of nonpermutation-repulsiveness and permutation-attractiveness. We prove that constraining a nonpermutation-repulsing automaton with a permutation-attracting constraint always reduces the state-space. This approach allows us to (i) compute optimal solutions of the BMO problem when the (additional) constraints are taken into account and (ii) compute bounds for the (original) BMO problem (without using the constraints). The approach is inspired by industrial practices, where manufacturing systems are typically over-specified [17] and in which over-specification is used implicitly and unconsciously to deal with complexity. Examples of overspecification encountered in industrial cases are disallowing multiple mapping possibilities for an operation or enforcing the static ordering of system operations. This approach enables system designers to consciously exploit over-specification by an explicit introduction of constraints to reduce the complexity of computing a solution to the BMO problem. We will demonstrate the 


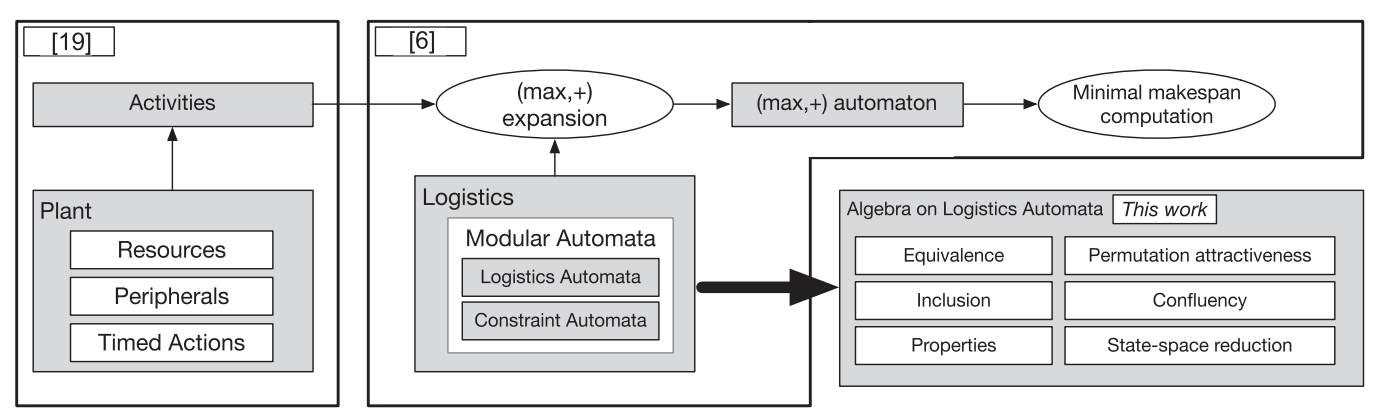

Fig. 1. Overview of ingredients from previous work that this article uses and extends upon.

(a)

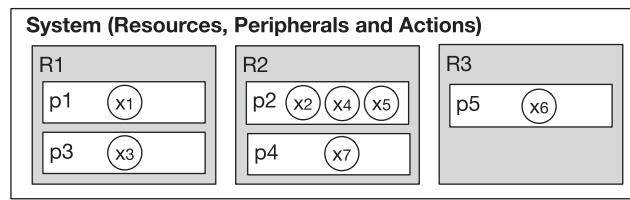

(c)

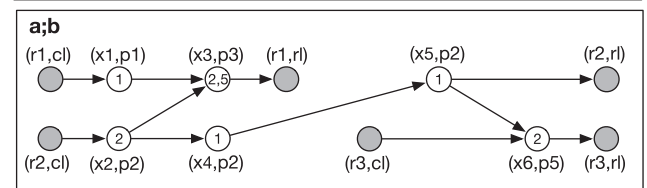

(b)

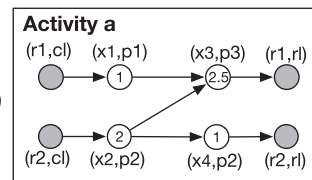

(d)

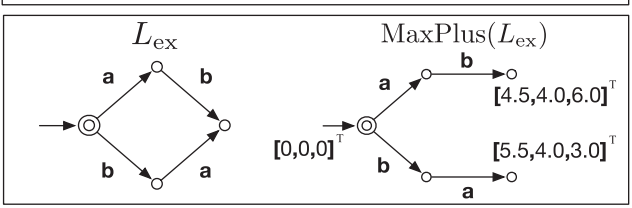

Fig. 2. Overview of the concepts of the framework of Reference [19]: (a) an FMS decomposed into resources R1, R2, and R3, peripherals p1, p2, p3, p4, and p5 and actions $x_{1}, x_{2}, x_{3}, x_{4}, x_{5}, x_{6}$, and $x_{7}$, (b) two example activities $a$ and $b$, (c) sequenced activity $a ; b$ and (d) example logistics automaton $L_{\mathrm{ex}}$ and (max,+) automaton $\operatorname{MaxPlus}\left(L_{\mathrm{ex}}\right)$.

effectiveness of our framework by specifying and optimizating an industrial wafer handling controller.

Overview: The remainder of this article is organized as follows: Sections 2 and 3 summarize the framework that we will extend in this article. In Section 4, we define equivalence and inclusion relations on logistics automata that take into account both behavioral and structural information, and we establish their key algebraic properties. Section 5 establishes sufficient conditions to ensure that automata constraining reduces state-space sizes. Section 6 demonstrates the use of the algebra to systematically relate automata languages, their state-space sizes, and their solutions to the BMO problem. In Section 7 the framework is applied to specify and optimize an industrial wafer handling controller. Related work is discussed in Section 8, and Section 9 concludes the article.

\section{SPECIFICATION FRAMEWORK}

This article extends the specification framework of Reference [19] (highlighted in Figure 1). In this section, we summarize the ingredients of this framework and its essential properties.

\subsection{Plant and Activities}

A manufacturing system is decomposed into a plant as a set of peripherals $(\mathcal{P})$, actions $(\mathcal{A})$, and resources $(\mathcal{R})$. Each peripheral can execute actions. An action describes an atomic behavior of the system, e.g., the movement of a motor or the actuation of an on/off peripheral such as a clamp. The complete set of actions describes all behavior that the system can exhibit. Peripherals are aggregated into resources, which can be claimed and released. Figure 2(a) depicts a synthetic example of 
a system decomposed into resources R1, R2, and R3, peripherals p1, p2, p3, p4, and p5 and actions $x_{1}, x_{2}, x_{3}, x_{4}, x_{5}, x_{6}$, and $x_{7}$. Using actions, deterministic functional behaviors of the system can be constructed as activities. An activity is a DAG, consisting of a set $N$ of nodes and a set $\rightarrow$ of dependencies between nodes. Nodes refer to either an action executed by a peripheral (associated with a pair $(x, p): x \in \mathcal{A}$ and $p \in \mathcal{P})$, or a claim (cl) or release (rl) of a resource (associated with a pair $(r, v): r \in \mathcal{R}$ and $v \in\{c l, r l\})$. Figure 2(b) depicts two such activities, $a$ and $b$. By sequencing multiple activities to form an activity sequence, we capture more elaborate operational behavior, such as the complete manufacturing of a product. We denote an activity sequence as $\underline{a}=a_{1} a_{2} \ldots a_{n}$ where $a_{1}, a_{2}, \ldots, a_{n}$ denote activities. The temporal behavior of an activity sequence can be determined by the sequencing operator (;) [19] that combines two activities in a new activity. Resource sharing and concurrency between multiple activities are taken into account by the correct claiming and releasing of resources between activities. Figure 2(c) depicts the result of sequencing activities $a$ and $b$, yielding $a ; b$. Intuitively, the releasing and claiming of shared resources must be correctly matched and replaced by a new dependency such that the resulting DAG is itself an activity.

Besides functional specification, the framework requires a temporal specification for performance optimization. To this end, a function $T: \mathcal{A} \rightarrow \mathbb{R}_{\geq 0}$ maps each action to its (fixed) execution time. Given an activity, the execution times can be lifted to the level of nodes, where the execution times of claim and releases nodes are assumed to be 0 . In Figures 2(b) and (c) the timing information is annotated within the nodes (except for the release and claim nodes). Since actions are executed on resources, a resource time stamp vector $\gamma_{R}: \mathcal{R} \rightarrow \mathbb{R}^{-\infty}$ represents the system state in terms of resource availability. For each $r \in \mathcal{R}, \gamma_{R}(r) \in \mathbb{R}^{-\infty}$ is the availability (here availability does not represent resource capacity, but timing availability) time of resource $r$. For example, in Figure 2(d), the initial system state is $\gamma_{R}=[0,0,0]^{T}$, implying that all system resources are available at time 0 .

Starting from resource vector $\gamma_{R}$ the execution of an activity $a$ leaves the system in a new state $\gamma_{R}^{\prime}$. This new state is determined by computing the completion times of each node in the activity, taking into account the dependencies and execution times of its nodes. In the example of Figure 2(d), the resource-time stamp vector after executing activities $a$ and $b$ is $\gamma_{R}^{\prime}=[4.5,4.0,6.0]^{T}$, thus for the next activity resources $R 1, R 2$, and $R 3$ are available at times $4.5,4.0$, and 6.0 , respectively.

It is well-known that $(\max ,+)$ algebra can be used to mathematically describe timed synchronous systems $[5,9,13]$. This observation also applies to activities and in Reference [19] it is shown that $\gamma_{R}^{\prime}=M_{a} \otimes \gamma_{R}$, where $M_{a}$ is the $(\max ,+)$ matrix of activity $a$ and where $\otimes$ is the $(\max ,+)$ matrix multiplication operator. In case a sequence $a_{1} a_{2} \cdots a_{n}$ of activities is executed, we thus have $\gamma_{R}^{\prime}=M_{a_{1} ; a_{2} ; \cdots ; a_{n}} \otimes \gamma_{R}$ (where ; denotes the activity sequencing operator). It is not difficult to see that this expression is equivalent to $M_{a_{n}} \otimes \cdots \otimes M_{a_{2}} \otimes M_{a_{1}} \otimes \gamma_{R}$. For a good introduction to $(\max ,+)$ algebra, we refer the reader to References $[1,5,15]$. To determine the makespan of an activity sequence, we assume the system to start with resource availability vector $\mathbf{0}_{R}$, i.e., for which $0_{R}(r)=0$ for each $r \in \mathcal{R}$. The makespan is determined by the $(\max ,+)$ norm of the state after execution. The makespan of activity sequence $\underline{a}=a_{1} a_{2} \cdots a_{n}$, written $m k s(\underline{a})$, is therefore given by $\left\|M_{a_{n}} \otimes \cdots \otimes M_{a_{2}} \otimes M_{a_{1}} \otimes 0_{R}\right\|$ (where $\|\cdot\|$ denotes the norm operator). As an example, we consider the sequenced activity $a ; b$ of Figure 2(c) again. The makespan of the corresponding activity sequence $a b$ is given by:

$$
m k s(a b)=M_{b} \otimes M_{a} \otimes\left[\begin{array}{l}
0 \\
0 \\
0
\end{array}\right]=\left[\begin{array}{ccc}
0 & -\infty & -\infty \\
-\infty & 1 & -\infty \\
-\infty & 3 & 2
\end{array}\right] \otimes\left[\begin{array}{ccc}
3.5 & 4.5 & -\infty \\
-\infty & 3 & -\infty \\
-\infty & -\infty & 0
\end{array}\right] \otimes\left[\begin{array}{l}
0 \\
0 \\
0
\end{array}\right]=\left[\begin{array}{c}
4.5 \\
4 \\
6
\end{array}\right]
$$

As a prelude to Section 2.1, Figure 2(d) presents a logistics automaton $L_{\text {ex }}$ encoding two activity sequences $a b$ and $b a$. To determine the activity sequences with the smallest makespan, the (max,+) 
automaton MaxPlus $\left(L_{\mathrm{ex}}\right)$ of $L_{\mathrm{ex}}$ is computed by decorating the states of $L_{\mathrm{ex}}$ with appropriate resource availability vectors. Activity sequences that terminate in states with the smallest vector norms are makespan-optimal, which is proven in Section 3. For the automaton $L_{\mathrm{ex}}$ in Figure 2(d) this happens to be sequence $b a$.

\subsection{Logistics}

An activity sequence can model the complete manufacturing of a product or batch of products, where a single activity models one manufacturing operation. In general, more than one activity sequence will satisfy the requirements imposed on the system. The set of all activity sequences that satisfy these requirements is encoded by a logistics automaton.

Definition 1 (Logistics Automaton). A logistics automaton is a tuple $\left\langle\mathcal{S}, \mathcal{A} c t, \dot{\rightarrow}, \mathcal{S}_{0}\right\rangle$, where $\mathcal{S}$ is a finite (possibly empty) set of states, $\mathcal{A} c t$ is a finite (possibly empty) set of activities, $\dot{\rightarrow} \subseteq \mathcal{S} \times \mathcal{A} c t \times$ $\mathcal{S}$ is a transition relation, and $\mathcal{S}_{0} \subseteq \mathcal{S}$ is a set of initial states. Here $\mathcal{S}_{0}=\left\{s_{0}\right\}$ except when $\mathcal{S}=\emptyset$. In that case the automaton is empty and therefore $\mathcal{S}_{0}=\emptyset$ as well. Let $s \stackrel{a}{\rightarrow} s^{\prime}$ be a shorthand for $\left(s, a, s^{\prime}\right) \in \dot{\rightarrow}$. The following additional properties must hold:

- there exists no $s \in \mathcal{S}$ such that $s \dot{\rightarrow}^{+} s$, where $\dot{\rightarrow}^{+}$is the transitive closure of $\dot{\rightarrow}$, and where $s \dot{\rightarrow} t$ denotes that $\stackrel{a}{\rightarrow} t$ for some $a \in \mathcal{A} c t$;

- if $\mathcal{S} \neq \emptyset$, then for all $s \in \mathcal{S}, s_{0} \dot{\rightarrow}^{*} s$, where $\dot{\rightarrow}^{*}$ is the reflexive transitive closure of $\rightarrow$.

A logistics automaton encodes a collection of activity sequences. This collection is called the language of the automaton.

Definition 2 (Language of a Logistics Automaton). Let $L=\left\langle\mathcal{S}, \mathcal{A} c t, \dot{\rightarrow}, \mathcal{S}_{0}\right\rangle$ be a logistics automaton. The language $\mathcal{L}(L)$ of $L$ is defined by

$$
\mathcal{L}(L)= \begin{cases}\emptyset, & \text { if } \mathcal{S}_{0}=\emptyset \\ \left\{\underline{a} \in \mathcal{A} c t^{*} \mid s_{0} \stackrel{a}{\rightarrow} s \text { for some } s \in \mathcal{S} \text { and } s \not{t}\right\}, & \text { if } \mathcal{S}_{0}=\left\{s_{0}\right\} .\end{cases}
$$

Here $\mathcal{A} c t^{*}$ denotes the collection of all sequences of activities in $\mathcal{A} c t$. Each $\underline{a} \in \mathcal{A} c t^{*}$ is of the form $a_{1} \ldots, a_{n}$, where $a_{i} \in \mathcal{A} c t(1 \leq i \leq n)$. For $n=0, \underline{a}$ is the empty activity sequence denoted by $\epsilon$. For states $s, s^{\prime} \in \mathcal{S}$ and $\underline{a}=a_{1}, \ldots, a_{n} \in \mathcal{A} c t^{*}$, we let $s \stackrel{a}{\rightarrow} s^{\prime}$ denote the existence of $s_{1}, \ldots, s_{n} \in \mathcal{S}$ such that $s \stackrel{a_{1}}{\longrightarrow} s_{1} \stackrel{a_{2}}{\longrightarrow} \cdots \stackrel{a_{n}}{\longrightarrow} s_{n}=s^{\prime}$. Further $s \stackrel{a}{\rightarrow}$ denotes that $s \stackrel{a}{\rightarrow} s^{\prime}$ for some $s^{\prime} \in \mathcal{S}, s \dot{a^{\prime}}$ denotes that $s \stackrel{a}{\rightarrow} s^{\prime}$ for some $a \in \mathcal{A} c t$ and $s^{\prime} \in \mathcal{S}$ and $s \not \rightarrow$ denotes that $s \rightarrow$ does not hold. Note that $\mathcal{L}(L)=\emptyset$ if $\mathcal{S}=\emptyset$ and $\mathcal{L}(L)=\{\epsilon\}$ if $\mathcal{S}=\left\{s_{0}\right\}$. Notice also that any sequence in the language should "run to completion." This means it should finish in a final state, i.e., a state with no outgoing transitions. As a consequence languages of logistics automata are not prefix closed in general.

Example 1. Recall Figure 2(d) depicting logistic automaton $L_{\mathrm{ex}}$. Nodes represent states and edges represent transitions. Activities are annotated on edges and the initial state is distinguished by an extra circumference. $L_{\mathrm{ex}}$ encodes the language $\mathcal{L}\left(L_{\mathrm{ex}}\right)=\{a b, b a\}$.

\subsection{Modular Logistics Specification: Composition and Constraining}

Even though a logistics automaton is able to encode the complete manufacturing of a batch of products, for large batch sizes or complex manufacturing jobs a monolithic automaton is not desired. For this purpose a composition operator on logistics automata is provided. The operator allows the manufacturing of the products to be specified individually and then composed to obtain an automata that encodes all the logistics requirements for the full batch of products. 
Definition 3 (Composition of Logistics Automata). Let $L_{1}=\left\langle\mathcal{S}_{1}, \mathcal{A} c t_{1}, \dot{\rightarrow}_{1}, S_{0_{1}}\right\rangle$ and $L_{2}=\left\langle\mathcal{S}_{2}, \mathcal{A c t}_{2}\right.$, $\left.\dot{\rightarrow}_{2}, S_{0_{2}}\right\rangle$ be logistics automata. Before we define the composition automaton $L_{1} \odot L_{2}$, we first define relation $\rightarrow \subseteq\left(\mathcal{S}_{1} \times \mathcal{S}_{2}\right) \times\left(\mathcal{A c t}_{1} \cup \mathcal{A} c t_{2}\right) \times\left(\mathcal{S}_{1} \times \mathcal{S}_{2}\right)$ as the smallest set $V$ satisfying the following inference rules:

$$
\begin{gathered}
\frac{s \stackrel{a}{\rightarrow}_{1} s^{\prime} \quad a \in \mathcal{A} c t_{1} \backslash \mathcal{A c t}_{2}}{(s, t) \stackrel{a}{\rightarrow}\left(s^{\prime}, t\right)}(1) \frac{s \stackrel{a}{\rightarrow}_{1} s^{\prime} \quad t \stackrel{a}{\rightarrow} t_{2} t^{\prime} \quad a \in \mathcal{A} c t_{1} \cap \mathcal{A c t}_{2}}{(s, t) \stackrel{a}{\rightarrow}\left(s^{\prime}, t^{\prime}\right)} \\
\frac{t \stackrel{a}{\rightarrow}_{2} t^{\prime} \quad a \in \mathcal{A} c t_{2} \backslash \mathcal{A} c t_{1}}{(s, t) \stackrel{a}{\rightarrow}\left(s, t^{\prime}\right)} \text { (3) }
\end{gathered}
$$

where $s, s^{\prime} \in \mathcal{S}_{1}$ and $t, t^{\prime} \in \mathcal{S}_{2}$. Now define the set of states $\mathcal{S}$ of the composition automaton as

$$
\mathcal{S}= \begin{cases}\emptyset, & \text { if } \mathcal{S}_{1}=\emptyset \text { or } \mathcal{S}_{2}=\emptyset \\ \left\{(s, t) \in \mathcal{S}_{1} \times \mathcal{S}_{2} \mid\left(s_{0_{1}}, s_{0_{2}}\right) \dot{\rightarrow}^{*}(s, t)\right. & \\ \text { and for some }\left(s^{\prime}, t^{\prime}\right) \in \mathcal{S}_{1} \times \mathcal{S}_{2} \text { with } & \\ \left.s^{\prime} \dot{\leftrightarrow}_{1} \text { and } t^{\prime} \dot{\phi}_{2},(s, t) \dot{\rightarrow}^{*}\left(s^{\prime}, t^{\prime}\right)\right\} & \text { if } \mathcal{S}_{0_{1}}=\left\{s_{0_{1}}\right\} \text { and } \mathcal{S}_{0_{2}}=\left\{s_{0_{2}}\right\} .\end{cases}
$$

Further define $\dot{\rightarrow}^{\prime}=\left\{\left((s, t), a,\left(s^{\prime}, t^{\prime}\right)\right) \subseteq \dot{\rightarrow} \mid(s, t),\left(s^{\prime}, t^{\prime}\right) \in \mathcal{S}\right\}$ and

$$
\mathcal{S}_{0}= \begin{cases}\emptyset, & \text { if } \mathcal{S}=\emptyset \\ \left\{\left(s_{0_{1}}, s_{0_{2}}\right)\right\} & \text { otherwise. }\end{cases}
$$

The composition automaton $L_{1} \odot L_{2}$ is now defined as $\left\langle\mathcal{S}, \mathcal{A} c t_{1} \cup \mathcal{A} c t_{2}, \dot{ }^{\prime}, \mathcal{S}_{0}\right\rangle$.

The language of a composite logistics automaton can be computed from the languages of its constituent automata by merging together the sequences of the languages of these constituents:

LEMMA 1. Let $L_{1}=\left\langle\mathcal{S}_{1}, \mathcal{A} c t_{1}, \dot{\rightarrow}_{1}, S_{0_{1}}\right\rangle$ and $L_{2}=\left\langle\mathcal{S}_{2}, \mathcal{A} c t_{2}, \dot{\rightarrow}_{2}, S_{0_{2}}\right\rangle$ be logistics automata. Then $\mathcal{L}\left(L_{1} \odot L_{2}\right)=\left\{\underline{a} \in\left(\mathcal{A c t}_{1} \cup \mathcal{A} c t_{2}\right)^{*} \mid \underline{a} \backslash \mathcal{A} c t_{1} \in \mathcal{L}\left(L_{1}\right)\right.$ and $\left.\underline{a} \backslash \mathcal{A c t}_{2} \in \mathcal{L}\left(L_{2}\right)\right\}$ where $\underline{a} \backslash \mathcal{A}_{c t} t_{1}$ and $\underline{a} \backslash \mathcal{A} c t_{2}$ denote the projections of activity sequence a onto alphabets $\mathcal{A c t}_{1}$ and $\mathcal{A c t}_{2}$, respectively.

By means of the composition operator, we can specify the behavior of a batch of products in a modular way. This operator respects the requirements specified for the individual products and ensures the completion of each of them. For a complete specification of the manufacturing of a batch of products, we also need to specify constraints across different product flows such as ordering constraints (e.g., FIFO ordering for a batch), safety constraints (e.g., access to exclusive safety areas), resource capacity constraints (e.g., a resource must be empty before receiving a product), or other constraints that are expressed as dependencies across different product flows. Such constraints are expressed in terms of constraint automata.

Definition 4 (Constraint Automaton). A constraint automaton is a tuple $\left\langle\mathcal{S}, \mathcal{A} c t, \dot{\rightarrow}, \mathcal{S}_{0}\right\rangle$, where $\mathcal{S}$ is a finite (possibly empty) set of states, $\mathcal{A} c t$ is a finite (possibly empty) set of activities, $\dot{\rightarrow} \subseteq$ $\mathcal{S} \times \mathcal{A} c t \times \mathcal{S}$ is a transition relation, and $\mathcal{S}_{0} \subseteq \mathcal{S}$ is a set of initial states, where $\mathcal{S}_{0}=\emptyset$ if $\mathcal{S}=\emptyset$ and $\mathcal{S}_{0}=\left\{s_{0}\right\}$ otherwise. The following additional property must hold:

- if $\mathcal{S} \neq \emptyset$, then for all $s \in \mathcal{S}, s_{0} \dot{\rightarrow}^{*} s$.

A constraint automaton encodes a language, just as a logistics automaton does.

Definition 5 (Language of a Constraint Automaton). Let $C=\left\langle\mathcal{S}, \mathcal{A} c t, \dot{\rightarrow}, \mathcal{S}_{0}\right\rangle$ be a constraint automaton. The language $\mathcal{L}(C)$ of $C$ is defined by

$$
\mathcal{L}(C)= \begin{cases}\emptyset, & \text { if } \mathcal{S}_{0}=\emptyset \\ \left\{\underline{a} \in \mathcal{A} c t^{*} \mid s_{0} \stackrel{a}{\rightarrow} s \text { for some } s \in \mathcal{S}\right\}, & \text { if } \mathcal{S}_{0}=\left\{s_{0}\right\} .\end{cases}
$$


Notice that constraint automata are distinct from logistics automata in the sense that they can be recursive and are therefore able to encode infinite languages. Constraints can be applied to logistics automata through the constraint operator. A constraint automaton $C=\left\langle\mathcal{S}_{2}, \mathcal{A c t}_{2}, \dot{\rightarrow}_{2}, \mathcal{S}_{0_{2}}\right\rangle$ is called a constraint on logistics automaton $L=\left\langle\mathcal{S}_{1}, \mathcal{A} c t_{1} \dot{\rightarrow}_{1}, \mathcal{S}_{0_{1}}\right\rangle$ if $\mathcal{A} c t_{2} \subseteq \mathcal{A} c t_{1}$. Applying constraint $C$ to automaton $L$ yields a new logistics automaton, which is denoted by $L \uparrow C$.

Definition 6 (Constraint Operator). Let $L=\left\langle\mathcal{S}_{1}, \mathcal{A} c t_{1}, \dot{\rightarrow}_{1}, \mathcal{S}_{0_{1}}\right\rangle$ be a logistics automaton and $C=$ $\left\langle\mathcal{S}_{2}, \mathcal{A} c t_{2}, \dot{\rightarrow}_{2}, \mathcal{S}_{0_{2}}\right\rangle$ be a constraint on L (so $\mathcal{A c t}_{2} \subseteq \mathcal{A} c t_{1}$ ). Before we define $L \uparrow C$, we first define relation $\dot{\subseteq} \subseteq\left(\mathcal{S}_{1} \times \mathcal{S}_{2}\right) \times \mathcal{A} c t_{l} \times\left(\mathcal{S}_{1} \times \mathcal{S}_{2}\right)$ as the smallest set $V$ satisfying the following inference rules:

$$
\frac{s \stackrel{a}{\rightarrow}_{1} s^{\prime} a \in \mathcal{A} c t_{1} \backslash \mathcal{A} c t_{2}}{(s, t) \stackrel{a}{\rightarrow}\left(s^{\prime}, t\right)} \text { (1) } \frac{s \stackrel{a}{\rightarrow}_{1} s^{\prime} t \stackrel{a}{\rightarrow} 2 t^{\prime} a \in \mathcal{A} c t_{1} \cap \mathcal{A} c t_{2}}{(s, t) \stackrel{a}{\rightarrow}\left(s^{\prime}, t^{\prime}\right)}
$$

where $s, s^{\prime} \in \mathcal{S}_{1}$ and $t, t^{\prime} \in \mathcal{S}_{2}$. Now define the set of states $\mathcal{S}$ of the constrained automaton as

$$
\mathcal{S}= \begin{cases}\emptyset, & \text { if } \mathcal{S}_{1}=\emptyset \text { or } \mathcal{S}_{2}=\emptyset \\ \left\{(s, t) \in \mathcal{S}_{1} \times \mathcal{S}_{2} \mid\left(s_{0_{1}}, s_{0_{2}}\right) \dot{\rightarrow}^{*}(s, t)\right. & \\ \text { and for some }\left(s^{\prime}, t^{\prime}\right) \in \mathcal{S}_{1} \times \mathcal{S}_{2} \text { with } & \\ \left.s^{\prime} \dot{\leftrightarrow}_{1},(s, t) \dot{\rightarrow}^{*}\left(s^{\prime}, t^{\prime}\right)\right\} & \text { if } \mathcal{S}_{0_{1}}=\left\{s_{0_{1}}\right\} \text { and } \mathcal{S}_{0_{2}}=\left\{s_{0_{2}}\right\} .\end{cases}
$$

Further define $\dot{\rightarrow}^{\prime}=\left\{\left((s, t), a,\left(s^{\prime}, t^{\prime}\right)\right) \subseteq \dot{\rightarrow} \mid(s, t),\left(s^{\prime}, t^{\prime}\right) \in \mathcal{S}\right\}$ and

$$
\mathcal{S}_{0}=\left\{\begin{array}{ll}
\emptyset, & \text { if } \mathcal{S}=\emptyset \\
\left\{\left(s_{0_{1}}, s_{0_{2}}\right)\right\} & \text { otherwise }
\end{array} .\right.
$$

The constrained automaton $L\left\lceil C\right.$ is now defined as $\left\langle\mathcal{S}, \mathcal{A} c t_{1}, \dot{\rightarrow}^{\prime}, \mathcal{S}_{0}\right\rangle$.

Both the constraint and composition operators assume multi-way synchronization for transitions. Note, however, that the constraint operator requires the logistics automaton to run to completion (i.e., reaches a final state), while this is not true for the constraint automaton. In other words, constraint automata capture only safety requirements (expressing that nothing bad should happen) while logistics automata capture both safety requirements and liveness requirements (expressing that something good happens eventually, namely, the completion of the different products in a batch). On the contrary, the composition operator requires both logistics automata to run to completion.

The language of a constrained logistics automaton can be computed from the languages of its constituent automata by filtering out activity sequences that are not consistent with the constraint:

Lemma 2. Let $L=\left\langle\mathcal{S}_{1}, \mathcal{A} c t_{1}, \dot{\rightarrow}_{1}, S_{0_{1}}\right\rangle$ be a logistics automaton and let $C=\left\langle\mathcal{S}_{2}, \mathcal{A c t}_{2}, \dot{\rightarrow}_{2}, S_{0_{2}}\right\rangle$ be a constraint on L. Then $\mathcal{L}\left(L\lceil C)=\left\{\underline{a} \in \mathcal{L}(L) \mid \underline{a} \backslash \mathcal{A} c t_{2} \in \mathcal{L}(C)\right\}\right.$.

Finally, we claim that the application of a constraint to a logistics automaton results in a subset of the original language:

Lemma 3 (LAnguage Constraining). Let $L$ be a logistics automaton and let $C$ be a constraint on L. Then $\mathcal{L}(L \uparrow C) \subseteq \mathcal{L}(L)$.

Example 2. Consider the automata depicted in Figure 3. $L_{1}$ and $L_{2}$ represent two distinct product flows and $L_{1} \odot L_{2}$ represents their combined execution flow in a two-product batch. Constraint $C_{1}$ represents a requirement on the order of activities $c$ and $d$ on the product modeled by $L_{1}$. Constraints $C_{2}$ and $C_{3}$ express requirements concerning the product flows of $L_{1} \odot L_{2}$. $\left(\left(L_{1} \odot L_{2}\right) \uparrow\right.$ $\left.C_{1}\right)\left\lceil C_{2}\right.$ shows the application of constraints $C_{1}$ and $C_{2}$ to $L_{1} \odot L_{2}$. Since $C_{1}$ requires activity $d$ to be preceded by $c$, transition $d$ is removed. Further, all behavior in which activity $e$ is not preceded by $a$ is removed due to $C_{2}$. $\left(\left(L_{1} \odot L_{2}\right) \uparrow C_{1}\right) \uparrow C_{3}$ shows the application of constraint $C_{1}$ and $C_{3}$ to $L_{1} \odot L_{2}$. 

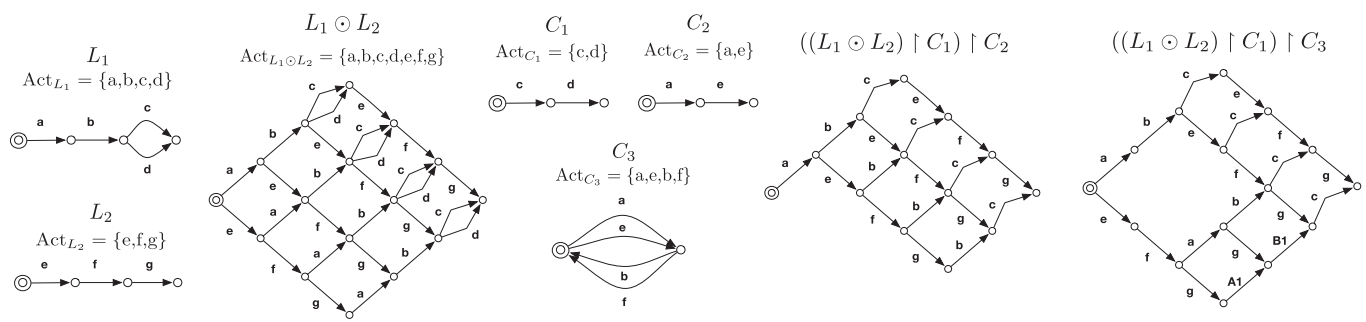

Fig. 3. Composing logistics automata $L_{1}$ and $L_{2}$ and constraining them with constraints $C_{1}, C_{2}$, and $C_{3}$.

In this case all behavior in which activities $b$ or $f$ and $a$ or $e$ do not occur in alternating order is removed (for instance sequence a e b c f g is removed). Notice that, consistent with Lemma 3, $\mathcal{L}\left(\left(\left(L_{1} \odot L_{2}\right)\left\lceil C_{1}\right)\left\lceil C_{2}\right) \subseteq \mathcal{L}\left(L_{1} \odot L_{2}\right)\right.\right.$ and $\mathcal{L}\left(\left(\left(L_{1} \odot L_{2}\right)\left\lceil C_{1}\right)\left\lceil C_{3}\right) \subseteq \mathcal{L}\left(L_{1} \odot L_{2}\right)\right.\right.$.

\section{BATCH MAKESPAN OPTIMIZATION}

We are interested in finding the activity sequence with the lowest makespan in the language of a logistics automaton. In Reference [6], we formalized this BMO problem and proved it to be NPhard:

Definition 7 (Batch Makespan Optimization (BMO)). Given a Logistics automaton $L$ determine an $\underline{a} \in \mathcal{L}(L)$ such that $m k s(\underline{a}) \leq m k s\left(\underline{a}^{\prime}\right)$, for all $\underline{a}^{\prime} \in \mathcal{L}(L)$

Lemma 4. The Batch Makespan Optimization problem is NP-hard.

Through language inclusion, we can establish sub-optimal solutions to the BMO problem and find bounds on the optimal makespan:

LEMmA 5. Let $L_{1}$ and $L_{2}$ be logistics automata for which $\mathcal{L}\left(L_{1}\right) \subseteq \mathcal{L}\left(L_{2}\right)$ and assume a to be a BMO solution to $L_{1}$ and $\underline{a}^{\prime}$ to be a BMO solution to $L_{2}$. Then $m k s(\underline{a}) \geq m k s\left(\underline{a}^{\prime}\right)$.

\subsection{Solving the BMO Problem: $(\max ,+)$ Automata}

The BMO problem can be solved in two steps. The first is to build a timed expansion of logistics automaton L using the $(\max ,+)$ characterization of its activities. The result of this expansion is a new logistics automaton where each state includes a resource time-stamp vector capturing the resource availability after executing the activities in the path leading to that state. Given a logistics automaton $L$, we call its timed expansion a $(\max ,+)$ automaton and denote it by $\operatorname{MaxPlus}(L)$.

Definition $8((\max ,+)$ automaton $)$. Let $L=\left\langle\mathcal{S}, \mathcal{A c t}, \dot{\rightarrow}, \mathcal{S}_{0}\right\rangle$ be a logistics automaton. First define $\operatorname{MaxPlusStates}(L)$ as the smallest set $V$ satisfying inference rules (1) and (2):

$$
\frac{\mathcal{S}_{0}=\left\{s_{0}\right\}}{\left(s_{0}, \mathbf{0}_{R}\right) \in V}(1) \frac{\left(s, \gamma_{R}\right) \in V \quad s \stackrel{a}{\rightarrow} s^{\prime}}{\left(s^{\prime}, \mathbf{M}_{a} \otimes \gamma_{R}\right) \in V}
$$

Here $\gamma_{R}$ denotes a resource time-stamp vector and $\mathbf{0}_{R}$ denotes the resource time-stamp vector containing only 0 valued entries. Note that $\mathbf{0}_{R}$ represents the initial system state in which all resources are available at time zero. $\mathbf{M}_{a}$ denotes the $(\max ,+)$ matrix corresponding to activity $a \in \mathcal{A} c t$ and $s, s^{\prime} \in \mathcal{S}$. Then, we define $\operatorname{MaxPlus}(L)$ as $\left(\operatorname{MaxPlusStates}(L), \mathcal{A} c t, \dot{\rightarrow}^{\prime}, \mathcal{S}_{0}^{\prime}\right)$, where $\rightarrow^{\prime}=$ $\left\{\left(s, \gamma_{R}\right), a,\left(s^{\prime}, \gamma_{R}^{\prime}\right) \in \operatorname{MaxPlusStates}(L) \times \mathcal{A} c t \times \operatorname{MaxPlusStates}(L) \mid s \stackrel{a}{\rightarrow} s^{\prime}\right.$ and $\left.\gamma_{R}^{\prime}=M_{a} \otimes \gamma_{R}\right\}$ and $\mathcal{S}_{0}^{\prime}=\emptyset$ if $\mathcal{S}_{0}=\emptyset$ and $\mathcal{S}_{0}^{\prime}=\left\{\left(s_{0}, \boldsymbol{0}_{R}\right)\right\}$ otherwise.

Each state of the logistics automaton can occur multiple times in the $(\max ,+)$ automaton, depending on the cumulative products of $(\max ,+)$ activity matrices along the paths from the initial state leading to this particular state. Therefore, the number of states of the $(\max ,+)$ automaton is 
at least as large as the number of states of the corresponding logistics automaton. Their languages are however equivalent, which is stated by the following lemma:

Lemma 6. Given a logistics automaton $L$ then $\mathcal{L}(L)=\mathcal{L}(\operatorname{MaxPlus}(L))$.

The state-space encoded by a $(\max ,+)$ automaton includes all the necessary functional and temporal information of the system in terms of allowed activity sequences as well as their respective completion times. For this reason, we denote the state-space of a $(\max ,+)$ automaton the optimization-space. The second step to solve the BMO problem is to explore all the final states (states with no outgoing transitions) of the optimization-space and compare the norms of the corresponding resource-time stamp vectors. Any sequence in the optimization-space leading to a final state with the lowest occurring norm is a solution to the BMO problem as stated in the following theorem:

Lemma 7. Let $L$ be a logistics automaton and $\operatorname{MaxPlus}(L)=\left\{\mathcal{S}, \dot{\rightarrow}, \mathcal{A c t}, \mathcal{S}_{0}\right\}$ its corresponding (max,+) automaton. Let $(s, \gamma) \in \mathcal{S}$ be such that $(s, \gamma) \nrightarrow$ and that for all $\left(s^{\prime}, \gamma^{\prime}\right) \in \mathcal{S}$ with $\left(s^{\prime}, \gamma^{\prime}\right) \nrightarrow$, $\|\gamma\| \leq\left\|\gamma^{\prime}\right\|$, and let $\underline{a} \in \mathcal{L}(L)$ be such that $\left(s_{0}, \mathbf{0}_{R}\right) \stackrel{a}{\rightarrow}(s, \gamma)$. Then for all $\underline{a}^{\prime} \in \mathcal{L}(L): m k s(\underline{a}) \leq$ $m k s\left(\underline{a}^{\prime}\right)$.

Example 3. Consider the $(\max ,+)$ automaton of logistics automaton $L_{\mathrm{ex}}$ depicted in Figure 2. Consistent with Lemma 6, indeed $\mathcal{L}\left(\operatorname{MaxPlus}\left(L_{\text {ex }}\right)\right)=\mathcal{L}\left(L_{\text {ex }}\right)$. The temporal execution of activity sequences $a b$ and $b a$ lead to different resource time-stamp vectors and thus the final state of $L_{\mathrm{ex}}$ is duplicated in MaxPlus $\left(L_{\text {ex }}\right)$. According to Lemma 7, activity sequences in $\mathcal{L}\left(L_{\text {ex }}\right)$ are makespanoptimal if they terminate in the states with the smallest vector norms in $\operatorname{MaxPlus}\left(L_{\mathrm{ex}}\right)$. The final state in MaxPlus $\left(L_{\mathrm{ex}}\right)$ with the smallest vector norm is labelled with vector $[5.5,4.0,3.0]^{T}$. This final state corresponds to sequence $b a$. Hence, $b a$ is a solution to the BMO problem of $L_{\mathrm{ex}}$.

\subsection{Worst-case Optimization-space: Tree Automata}

The size of the state-space of a (max,+) automaton is at least as large as that of the corresponding logistics automaton, a fact that we will prove in the next section. It can even grow exponentially in the size of the state-space of the corresponding logistics automaton, which is the very reason that the BMO problem is NP-hard. In the worst case each path in the logistics automaton induces a unique cumulative product of $(\max ,+)$ matrices. This worst case is captured by what we will call the tree automaton $\operatorname{Tree}(L)$ of $L$, the proof of which is given in the next section.

Definition 9 (Tree Automaton). Let $L=\left\langle\mathcal{S}, \mathcal{A} c t, \dot{\rightarrow}, \mathcal{S}_{0}\right\rangle$ be a logistics automaton. We first define Paths $(L)$ as the smallest set $V$ satisfying:

$$
\frac{\mathcal{S}_{0}=\left\{s_{0}\right\}}{s_{0} \in V}(1) \quad \frac{q s \in V \quad s \stackrel{a}{\rightarrow} s^{\prime}}{q s a s^{\prime} \in V}
$$

Here $s, s^{\prime} \in \mathcal{S}$ and $a \in \mathcal{A} c t$. Paths $(L)$ contains sequences of elements in $\mathcal{S}$ and $\mathcal{A} c t$. Each sequence is of the form $s_{0} a_{0} s_{1} a_{1} \cdots s_{n}$ and encodes the path from starting state $s_{0}$ to state $s_{n}$ (via transitions labeled with activities $a_{0} \cdots a_{n-1}$ and intermediate states $s_{1} \cdots s_{n-1}$ ). In inference rule (2), $q s$ refers to a path that ends in state $s$. Note that $q$ can refer to an empty sequence of elements. Remark that Paths $(L)=\emptyset$ if $\mathcal{S}=\emptyset$. We now define Tree $(L)$ as $\left(\operatorname{Paths}(L), \mathcal{A} c t, \stackrel{\sim}{\rightarrow}^{\prime}, \mathcal{S}_{0}^{\prime}\right)$, where $\dot{\check{\rightarrow}}^{\prime}=\left\{\left(q s, a, q s a s^{\prime}\right) \in \operatorname{Paths}(L) \times \mathcal{A} c t \times \operatorname{Paths}(L) \mid s \stackrel{a}{\rightarrow} s^{\prime}\right\}$ and $\mathcal{S}_{0}^{\prime}=\emptyset$ if $\mathcal{S}_{0}=\emptyset$ and $\mathcal{S}_{0}^{\prime}=\left\{s_{0}\right\}$ otherwise.

Just like the MaxPlus operator, the Tree operator leaves the language of the logistics automaton unaltered.

LEMMA 8. Given a logistics automaton $L$ then $\mathcal{L}(L)=\mathcal{L}($ Tree $(L))$. 


\section{ALGEBRA ON LOGISTICS AUTOMATA}

So far, we introduced the $\odot, \uparrow$, MaxPlus, and Tree operators on logistics automata. In this section, we complement these operators with equivalence and inclusion relations and prove important algebraic properties. In this fashion, we define an algebra on logistics automata to systematically relate their languages, their state-space sizes, and their optimization-space sizes.

\subsection{Equivalence and Inclusion Relations}

We start by defining equivalence and inclusion relations on logistics automata that capture both behavioral and structural aspects. The behavioral aspect relates the languages of the automata, while the structural aspect relates their state-space sizes.

Definition 10 (Equivalence). Let $L_{1}=\left\langle\mathcal{S}_{1}, \mathcal{A} c t_{1}, \dot{\rightarrow}_{1}, \mathcal{S}_{0_{1}}\right\rangle$ and $L_{2}=\left\langle\mathcal{S}_{2}, \mathcal{A} c t_{2}, \dot{\rightarrow}_{2}, \mathcal{S}_{0_{2}}\right\rangle$ be logistics automata. Then $L_{1}$ and $L_{2}$ are equivalent, written $L_{1} \approx L_{2}$, if and only if $\mathcal{A}_{c} t_{1}=\mathcal{A} c t_{2}$ and either (i) $\mathcal{S}_{1}=\emptyset$ and $\mathcal{S}_{2}=\emptyset$ or (ii) $\mathcal{S}_{0_{1}}=\left\{s_{0_{1}}\right\}$ and $\mathcal{S}_{0_{2}}=\left\{s_{0_{2}}\right\}$ and there exists a bijective function $\mathcal{F}: \mathcal{S}_{1} \rightarrow$ $\mathcal{S}_{2}$ satisfying:

(1) $\mathcal{F}\left(s_{0_{1}}\right)=s_{0_{2}}$;

(2) For all $s, s^{\prime} \in \mathcal{S}_{1}$ and $a \in \mathcal{A} c t_{1} s \stackrel{a}{\rightarrow} s^{\prime}$ if and only if $F(s) \stackrel{a}{\rightarrow} F\left(s^{\prime}\right)$.

THEOREM $1 . \approx$ is an equivalence relation on logistics automata.

Equivalent logistics automata encode the same languages, as stated in the following lemma:

Lemma 9. Let $L_{1}$ and $L_{2}$ be equivalent logistics automata. Then $\mathcal{L}\left(L_{1}\right)=\mathcal{L}\left(L_{2}\right)$.

In correspondence to the equivalence relation, we define a partial order (inclusion) relation on logistics automata.

Definition 11 (Inclusion). Let $L_{1}=\left\langle\mathcal{S}_{1}, \mathcal{A} c t_{1}, \dot{\rightarrow}_{1}, \mathcal{S}_{0_{1}}\right\rangle$ and $L_{2}=\left\langle\mathcal{S}_{2}, \mathcal{A} c t_{2}, \dot{\rightarrow}_{2}, \mathcal{S}_{0_{2}}\right\rangle$ be logistics automata. Then $L_{1}$ is included in $L_{2}$, written $L_{1} \sqsubseteq L_{2}$, if and only if $\mathcal{A} c t_{1}=\mathcal{A} c t_{2}$ and either (i) $\mathcal{S}_{1}=\emptyset$ or (ii) $\mathcal{S}_{0_{1}}=\left\{s_{0_{1}}\right\}$ and $\mathcal{S}_{0_{2}}=\left\{s_{0_{2}}\right\}$ and there exists an injective relation $R \subseteq \mathcal{S}_{1} \times \mathcal{S}_{2}$ satisfying:

(1) $\left(s_{0_{1}}, s_{0_{2}}\right) \in R$;

(2) For all $\left(s_{1}, s_{2}\right) \in R$ and $a \in \mathcal{A} c t_{1}$ if $s_{1} \stackrel{a}{\rightarrow}{ }_{1} s_{1}^{\prime}$ (for some $s_{1}^{\prime} \in \mathcal{S}_{1}$ ) then $s_{2} \stackrel{a}{\rightarrow}{ }_{2} s_{2}^{\prime}$ (for some $\left.s_{2}^{\prime} \in \mathcal{S}_{2}\right)$ and $\left(s_{1}^{\prime}, s_{2}^{\prime}\right) \in R$

(3) For all $\left(s_{1}, s_{2}\right) \in R$ if $s_{2} \dot{\rightarrow}_{2}$, then $s_{1} \dot{\rightarrow}_{1}$.

THEOREM 2 . $\sqsubseteq$ is a partial order relation on logistics automata. In particular $L_{1} \approx L_{2}$ if and only if $L_{1} \sqsubseteq L_{2}$ and $L_{2} \sqsubseteq L_{1}$.

If a logistics automaton is included in another logistics automaton, the size of the state-space of the former never exceeds that of the latter.

Lemma 10. Let $L_{1}=\left\langle\mathcal{S}_{1}, \mathcal{A} c t_{1}, \dot{\rightarrow}_{1}, \mathcal{S}_{0_{1}}\right\rangle$ and $L_{2}=\left\langle\mathcal{S}_{2}, \mathcal{A} c t_{2}, \dot{\rightarrow}_{2}, \mathcal{S}_{0_{2}}\right\rangle$ be logistics automata such that $L_{1} \sqsubseteq L_{2}$. Then \# $\mathcal{S}_{1} \leq \# \mathcal{S}_{2}$, where operator \# counts the number of states.

Example 4. Consider logistics automata $L_{h}$ and $L_{i}$ depicted in Figure 4 . It is easy to check that $L_{i}$ is included in $L_{h}$, but $L_{h}$ is not included in $L_{i} . L_{h}$ and $L_{i}$ are thus not equivalent, even though they have the same language. Note that our inclusion relation is stronger than Milner's [14] simulation relation $\prec$ for which both $L_{h} \prec L_{i}$ and $L_{i}<L_{h}$ hold. $\prec$ is a pre-order but not a partial order in the sense that $L_{i} \nsim L_{h}$, where $\sim$ denotes Milner's strong bisimulation. The essential property that makes $\sqsubseteq$ into a partial order is the property of injectivity (see Definition 11). Consider logistics automata $L_{j}$ and $L_{k}$ depicted in Figure 4. Even though $L_{j} \prec L_{k}$ holds, we have $L_{j} \nsubseteq L_{k}$. This is 

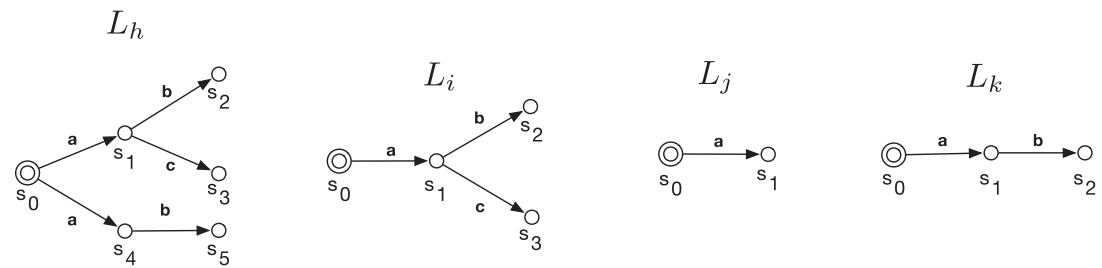

Fig. 4. Example where logistics automaton $L_{i}$ is included in $L_{h}$ but $L_{h}$ is not included in $L_{i}$; and logistics automaton $L_{j}$ is not included in logistics automaton $L_{k}$.

because the inclusion relation forces (by condition 3 of Definition 11) all activity sequences of $\mathcal{L}\left(L_{j}\right)$ to be included in $\mathcal{L}\left(L_{k}\right)$.

\subsection{Algebraic Properties}

We defined an algebra on (logistics and constraint) automata with composition $(\odot)$, constraining $(\uparrow)$, MaxPlus, and Tree operators, together with a partial-order $(\subseteq, \subseteq)$ and equivalence relations $(\approx$, $=)$. The algebra satisfies several properties of which the importance is demonstrated in Section 6 .

Theorem 3. Let $L_{1}, L_{2}$, and $L_{3}$ be logistics automata and let $C_{1}$ and $C_{2}$ be constraints. Then:

1. $L_{1} \odot L_{2} \approx L_{2} \odot L_{1}($ commutativity $\odot)$.

2. $\left(L_{1} \odot L_{2}\right) \odot L_{3} \approx L_{1} \odot\left(L_{2} \odot L_{3}\right)$ (associativity $\left.\odot\right)$.

3. $\left(L_{1}\left\lceil C_{1}\right)\left\lceil C_{2} \approx\left(L_{1}\left\lceil C_{2}\right) \uparrow C_{1}\right.\right.\right.$ if $C_{1}$ and $C_{2}$ are constraints on $L_{1}$ (commutativity $\left.\uparrow\right)$.

4. $\left(L_{1} \odot L_{2}\right)\left\lceil C_{1} \approx\left(L_{1}\left\lceil C_{1}\right) \odot\left(L_{2}\left\lceil C_{1}\right)\right.\right.\right.$ if $C_{1}$ is a constraint on both $L_{1}$ and $L_{2}$ (distributivity †).

5. $L_{1} \sqsubseteq L_{2}$ implies $L_{1} \odot L \sqsubseteq L_{2} \odot L$ (substitutivity $\sqsubseteq$ under $\left.\odot\right)$.

6. $L_{1} \approx L_{2}$ implies $L_{1} \odot L \approx L_{2} \odot L$ (substitutivity $\approx$ under $\odot$ ).

7. $\mathcal{L}\left(L_{1}\right) \subseteq \mathcal{L}\left(L_{2}\right)$ implies $\mathcal{L}\left(L_{1} \odot L_{3}\right) \subseteq \mathcal{L}\left(L_{2} \odot L_{3}\right)$ (substitutivity $\subseteq$ under $\left.\odot\right)$.

8. $\mathcal{L}\left(L_{1}\right)=\mathcal{L}\left(L_{2}\right)$ implies $\mathcal{L}\left(L_{1} \odot L_{3}\right)=\mathcal{L}\left(L_{2} \odot L_{3}\right)$ (substitutivity $=$ under $\left.\odot\right)$.

9. $L_{1} \sqsubseteq L_{2}$ implies $L_{1}\left\lceil C_{1} \sqsubseteq L_{2}\left\lceil C_{1}\right.\right.$ if $C_{1}$ is a constraint on $L_{1}$ and $L_{2}$ (substitutivity $\sqsubseteq$ under $\uparrow$ ).

10. $L_{1} \approx L_{2}$ implies $L_{1}\left\lceil C_{1} \approx L_{2} \uparrow C_{1}\right.$ if $C_{1}$ is a constraint on $L_{1}$ and $L_{2}$ (substitutivity $\approx$ under $\uparrow$ ).

11. $\mathcal{L}\left(L_{1}\right) \subseteq \mathcal{L}\left(L_{2}\right)$ implies $\mathcal{L}\left(L_{1}\left\lceil C_{1}\right) \subseteq \mathcal{L}\left(L_{2}\left\lceil C_{1}\right)\right.\right.$ if $C_{1}$ is a constraint on $L_{1}$ and $L_{2}$ (substitutivity $\subseteq$ under \lceil) .

12. $\mathcal{L}\left(L_{1}\right)=\mathcal{L}\left(L_{2}\right)$ implies $\mathcal{L}\left(L_{1}\left\lceil C_{1}\right)=\mathcal{L}\left(L_{2}\left\lceil C_{1}\right)\right.\right.$ if $C_{1}$ is a constraint on $L_{1}$ and $L_{2}$ (substitutivity $=$ under \lceil) .

13. $L_{1} \sqsubseteq L_{2}$ implies MaxPlus $\left(L_{1}\right) \sqsubseteq \operatorname{MaxPlus}\left(L_{2}\right)$ (substitutivity $\sqsubseteq$ under MaxPlus).

14. $L_{1} \approx L_{2}$ implies MaxPlus $\left(L_{1}\right) \approx \operatorname{MaxPlus}\left(L_{2}\right)$ (substitutivity $\approx$ under MaxPlus).

15. $L_{1} \sqsubseteq L_{2}$ implies Tree $\left(L_{1}\right) \sqsubseteq \operatorname{Tree}\left(L_{2}\right)$ (substitutivity $\sqsubseteq$ under Tree).

16. $L_{1} \approx L_{2}$ implies Tree $\left(L_{1}\right) \approx \operatorname{Tree}\left(L_{2}\right)$ (substitutivity $\approx$ under Tree).

17. $\mathcal{L}\left(L_{1}\right) \subseteq \mathcal{L}\left(L_{2}\right)$ implies $\mathcal{L}\left(\operatorname{MaxPlus}\left(L_{1}\right)\right) \subseteq \mathcal{L}\left(\operatorname{MaxPlus}\left(L_{2}\right)\right)$ (substitutivity $\subseteq$ under MaxPlus).

18. $\mathcal{L}\left(L_{1}\right)=\mathcal{L}\left(L_{2}\right)$ implies $\mathcal{L}\left(\operatorname{MaxPlus}\left(L_{1}\right)\right)=\mathcal{L}\left(\operatorname{MaxPlus}\left(L_{2}\right)\right)$ (substitutivity $=$ under MaxPlus).

19. $\mathcal{L}\left(L_{1}\right) \subseteq \mathcal{L}\left(L_{2}\right)$ implies $\mathcal{L}\left(\right.$ Tree $\left.\left(L_{1}\right)\right) \subseteq \mathcal{L}\left(\right.$ Tree $\left.\left(L_{2}\right)\right)$ (substitutivity $\subseteq$ under Tree).

20. $\mathcal{L}\left(L_{1}\right)=\mathcal{L}\left(L_{2}\right)$ implies $\mathcal{L}\left(\right.$ Tree $\left.\left(L_{1}\right)\right)=\mathcal{L}\left(\right.$ Tree $\left.\left(L_{2}\right)\right)$ (substitutivity $=$ under Tree).

21. $L_{1} \sqsubseteq \operatorname{MaxPlus}\left(L_{1}\right) \sqsubseteq \operatorname{Tree}\left(L_{1}\right)$ (optimization-space bounds).

22. $\mathcal{L}\left(L_{1}\right)=\mathcal{L}\left(\operatorname{MaxPlus}\left(L_{1}\right)\right)=\mathcal{L}\left(\right.$ Tree $\left.\left(L_{1}\right)\right)$ (MaxPlus and Tree preserve language).

23. $L_{1} \sqsubseteq L_{2}$ implies $\mathcal{L}\left(L_{1}\right) \subseteq \mathcal{L}\left(L_{2}\right)$ (巨 implies $\subseteq$ ).

24. $L_{1} \approx L_{2}$ implies $\mathcal{L}\left(L_{1}\right)=\mathcal{L}\left(L_{2}\right)(\approx$ implies $=)$. 


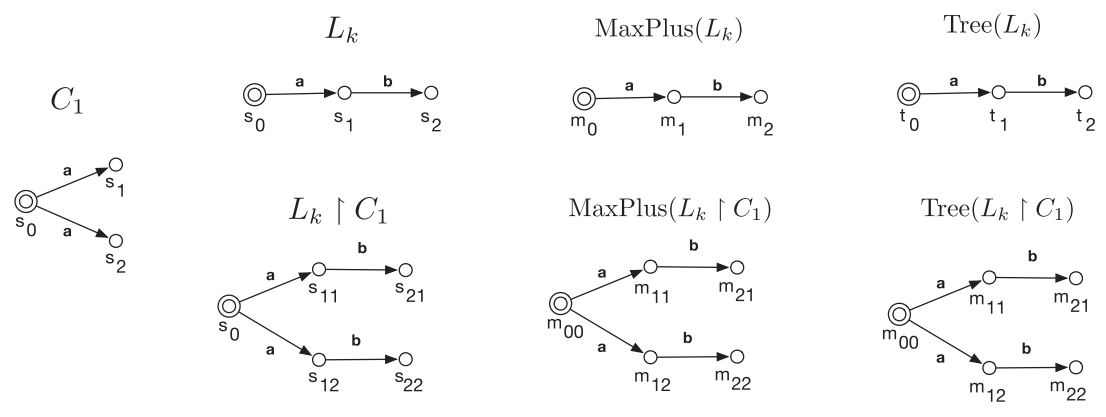

Fig. 5. The constraining of $L_{k}$ with a non-deterministic constraint $C_{1}$ results in a constrained automaton $L_{k} \uparrow C$ that is not included in $L_{k}$. The same is true for their $(\max ,+)$ and tree counterparts.

\section{OPTIMIZATION-SPACE REDUCTION}

In the previous section, we introduce an algebra on logistics automata that is able to relate their languages and state-space sizes. In this section, we discuss how to apply this algebra to reduce ${ }^{1}$ the optimization-space by constraining logistics automata. For this purpose, we establish sufficient conditions on $L$ and $C$ so $L \uparrow C \sqsubseteq L$. First recall from Lemma 3 that $\mathcal{L}(L \uparrow C) \subseteq \mathcal{L}(L)$. This does not imply, however, that $L \uparrow C$ is included in $L$. In general, even $\operatorname{MaxPlus}(L \uparrow C)$ is not included in $\operatorname{MaxPlus}(L)$ and Tree $(L\lceil C)$ is not included in Tree $(L)$. This implies that constraining does not in general lead to optimization-space reduction, not even a reduction of the worst-case optimizationspace. This is demonstrated in Example 5.

Example 5. Consider logistics automaton $L_{k}$, constraint automaton $C_{1}$, and constrainted automaton $L_{k}\left\lceil C_{1}\right.$ depicted in Figure 5. Obviously $L_{k}\left\lceil C_{1} \nsubseteq L_{k}\right.$, MaxPlus $\left(L_{k}\lceil C) \nsubseteq \operatorname{MaxPlus}\left(L_{k}\right)\right.$ and Tree $\left(L_{k}\lceil C) \nsubseteq \operatorname{Tree}\left(L_{k}\right)\right.$. This is caused by constraint $C_{1}$ that is non-deterministic.

Example 5 shows that non-deterministic constraints can increase the worst-case optimization space (which is the state-space of the Tree automaton). However, if a constraint is deterministic this worst-case optimization space will not increase, which follows from the following lemma:

LEMmA 11. Let $L=\left\langle\mathcal{S}_{1}, \mathcal{A} c t_{1}, \dot{\rightarrow}_{1}, S_{0_{1}}\right\rangle$ be a logistics automaton and $C=\left\langle\mathcal{S}_{2}, \mathcal{A c t}_{2}, \dot{\rightarrow}_{2}, S_{0_{2}}\right\rangle$ a constraint on L. IfC is deterministic, then Tree $(L\lceil C) \sqsubseteq \operatorname{Tree}(L)$.

Example 6. Consider logistics automaton $L_{m}$, constraint $C_{2}$, and constrained automaton $L_{m} \uparrow C_{2}$ depicted in Figure 6 . Since $C_{2}$ is deterministic, we have Tree $\left(L_{m}\left\lceil C_{2}\right) \sqsubseteq \operatorname{Tree}\left(L_{m}\right)\right.$ (consistent with Lemma 11). However, we also see that $L_{m}\left\lceil C_{2} \nsubseteq L_{m}\right.$ and that $\operatorname{MaxPlus}\left(L_{m}\left\lceil C_{2}\right) \nsubseteq \operatorname{MaxPlus}\left(L_{m}\right)\right.$. Notice in particular that constraining led to an increased size of the $(\max ,+)$ state-space.

\subsection{Nonpermutation-repulsing and Permutation-attracting Automata}

From Lemma 11 and Example 6, we learn that deterministic constraints lead to a reduction of the worst-case optimization-space. Unfortunately, the optimization-space itself may still grow when deterministic constraints are applied. To achieve our goal in reducing the optimization-space, we recall Theorem 3.13 stating that $L\lceil C \sqsubseteq L$ implies $\operatorname{MaxPlus}(L \uparrow C) \sqsubseteq \operatorname{MaxPlus}(L)$. We thus want to make sure that $L\lceil C \sqsubseteq L$. In the remainder of this section, we establish sufficient conditions (on $L$ and $C$ ) for this to be true. We start by defining the two fundamental properties of automata.

\footnotetext{
${ }^{1}$ With state-space reduction, we mean that the state-space will not grow.
} 


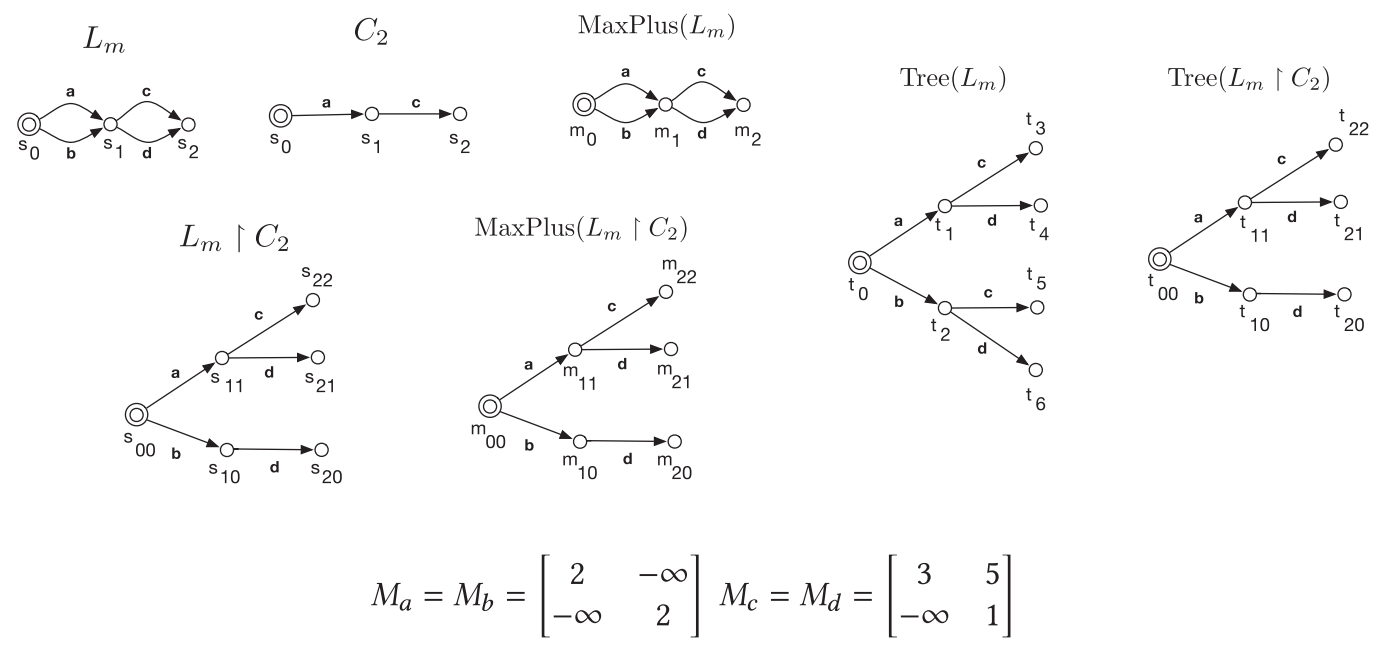

Fig. 6. Example showing that in the case of a deterministic constraint $C_{2}$ the tree of the constrained automaton (Tree $\left(L_{m}\left\lceil C_{2}\right)\right)$ is included in tree of the logistics automaton (Tree $\left.\left(L_{m}\right)\right)$, while this inclusion does in general neither hold for the constrained automaton nor for the MaxPlus automaton.

Definition 12 (Nonpermutation-Repulsing). Let $L C=\left\langle\mathcal{S}, \mathcal{A} c t, \dot{\rightarrow}, \mathcal{S}_{0}\right\rangle$ be either a logistics automaton or a constraint. Then $L C$ is called nonpermutation-repulsing (or np-repulsing for short) if either $\mathcal{S}=\emptyset$ or $\mathcal{S}=\left\{s_{0}\right\}$ and the following condition holds:

(1) For all $s \in \mathcal{S}$ and $\underline{a}, \underline{a}^{\prime} \in \mathcal{A} c t^{*}$, if $s_{0} \stackrel{a}{\rightarrow} s$ and $s_{0} \stackrel{\underline{a}^{\prime}}{\rightarrow} s$ then $\underline{a} \sim^{p} \underline{a}^{\prime}$.

Here $\underline{a} \sim^{p} \underline{a}^{\prime}$ denotes that activity sequences $\underline{a}$ and $\underline{a}^{\prime}$ are permutations.

Intuitively, an automaton is np-repulsing if all sequences running to a certain state are permutations of one another. This state "repulses" sequences that are not permutations (of the sequences that lead to this state). Notice that permuting sequences do not necessarily lead to the same state. In case they do, we call the automaton permutation-attracting:

Definition 13 (Permutation-Attracting). Let $L C=\left\langle\mathcal{S}, \mathcal{A c t}, \dot{\rightarrow}, \mathcal{S}_{0}\right\rangle$ be either a logistics automaton or a constraint. Then $L C$ is called permutation-attracting (or p-attracting for short) if either $\mathcal{S}=\emptyset$ or $\mathcal{S}=\left\{s_{0}\right\}$ and the following condition holds:

(1) For all $s, s^{\prime} \in \mathcal{S}$ and $\underline{a}, \underline{a}^{\prime} \in \mathcal{A} c t^{*}$ with $\underline{a} \sim^{p} \underline{a}^{\prime}$, if $s_{0} \stackrel{a}{\rightarrow} s$ and $s_{0} \stackrel{\underline{a}^{\prime}}{\rightarrow} s^{\prime}$ then $s=s^{\prime}$.

Thus, all permuting sequences in the language of a p-attracting automaton lead to the same state. This single state "attracts" all these permutations, so to speak.

Example 7. Consider the automata $L_{q}, L_{r}, L_{s}$, and $L_{t}$ of Figure 7. $L_{q}$ is neither np-repulsing nor p-attracting. When leaving out the transition from the initial state to state $s_{2}$, we are left with automaton $L_{r}$, which is p-attracting but not np-repulsing, since both sequences $a$ and $b$ lead to state $s_{1}$. Automaton $L_{s}$, however, is np-repulsing but not p-attracting. If states $s_{4}$ and $s_{5}$ are removed from $L_{s}$, we are left with $L_{t}$, which is both np-repulsing and p-attracting.

Notice that the notions of np-repulsiveness and p-attractiveness are independent. Interestingly, both notions are preserved by the composition operator:

Lemma 12. Let $L_{1}$ and $L_{2}$ be logistics automata. If $L_{1}$ and $L_{2}$ are both np-repulsing, then so is $L_{1} \odot L_{2}$. If $L_{1}$ and $L_{2}$ are both p-attracting, then so is $L_{1} \odot L_{2}$. 

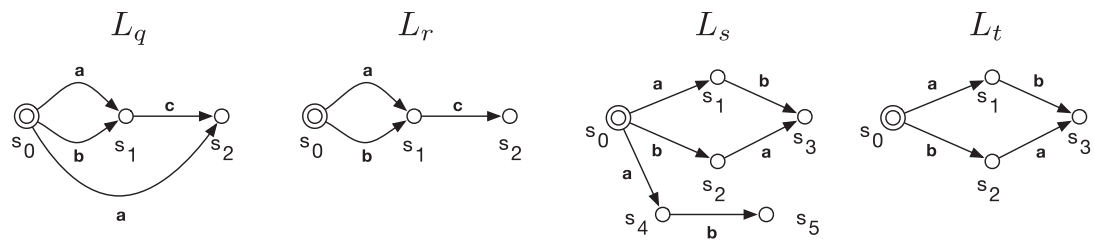

Fig. 7. Examples: $L_{q}$ is neither np-repulsing nor p-attracting. $L_{r}$ is p-attracting but not np-repulsing. $L_{s}$ is np-repulsing but not $\mathrm{p}$-attracting. $L_{t}$ is both $\mathrm{np}$-repulsing and p-attracting.
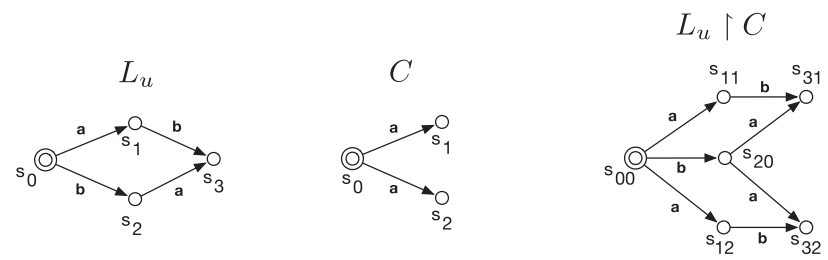

Fig. 8. Np-repulsiveness is preserved by constraining while p-attractiveness is not as shown by $L_{u}\lceil C$.

We further have that np-repulsiveness is preserved by general constraining:

Lemma 13. Let $L$ be an np-repulsing logistics automata and let $C$ be any constraint. Then $L \uparrow C$ is np-repulsing.

Example 8. Consider automaton $L_{u}$ and constraint $C$ depicted in Figure 8. $L_{u}$ is both nprepulsing and p-attracting and $C$ is nondeterministic. Constrained automaton $L_{u}\lceil C$ is nprepulsing (consistent with Lemma 13), but not p-attracting.

From the previous example, we learn that $\mathrm{p}$-attractiveness is not preserved by general constraining. However, if both the logistics automaton and the constraint are p-attractive, the constrained automaton is p-attractive as well, which is stated in the following lemma:

LEMMA 14. Let $L$ be a p-attracting logistics automaton and let $C$ be a p-attracting constraint. Then $L \uparrow C$ is p-attracting.

Example 9. Consider again automaton $L_{u}$ and constraint $C$ depicted in Figure 8. Since $C$ is nondeterministic it is not p-attracting. Removing any of the nondeterministic branches from $C$ would render both the constraint and the constrained automaton p-attracting.

We now arrive at the main theorem of this article. It establishes sufficient conditions on $L$ and $C$ for $L \uparrow C \sqsubseteq L$ to hold, thereby ensuring the constraining to imply optimization-space reduction.

Theorem 4. Let $L$ be an np-repulsing logistics automata and let $C$ be a p-attracting constraint. Then $L \uparrow C \sqsubseteq L$.

Example 10. Consider the np-repulsing logistics automaton $L_{v}$ and the p-attracting constraint $C$ as shown in Figure 9. Consistent with Theorem 4, we observe that $L_{v}\left\lceil C \sqsubseteq L_{v}\right.$. Further, from Lemmas 3.13 and 3.15, we know that both $\operatorname{MaxPlus}\left(L_{v}\lceil C) \sqsubseteq \operatorname{MaxPlus}\left(L_{v}\right)\right.$ and $\operatorname{Tree}\left(L_{v}\lceil C) \sqsubseteq\right.$ $\operatorname{Tree}\left(L_{v}\right)$. Consequently, from Lemma 10 , we know that the both the $(\max ,+)$ optimization space and the worst-case optimization space are reduced, which is also obvious from Figure 9.

The reader may have wondered about the relations between np-repulsiveness and pattractiveness and the well-known concept of confluence. Indeed, the automaton $L_{v}$ depicted in Figure 9 is confluent, since it has the so-called diamond property. In the remainder of this section, we will show that a confluent logistics automaton is both np-repulsing and p-attracting, but 


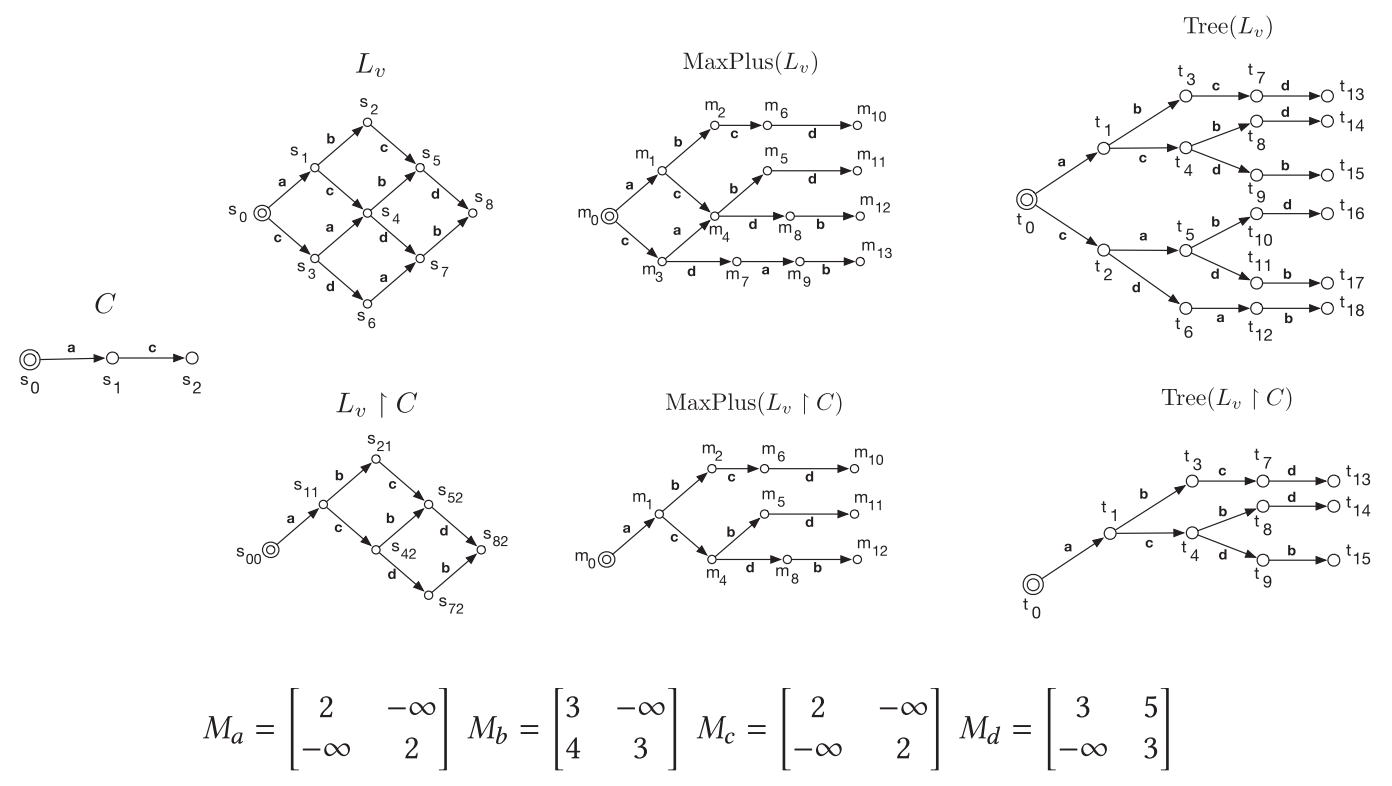

Fig. 9. Constraining an np-repulsing automaton with a p-attracting constraint reduces the $(\max ,+)$ optimization-space as well as the worst-case optimization-space.

not vice versa. We will also show that a confluent constraint automaton is p-attracting, but not necessarily np-repulsing. If a confluent constraint is non-recursive, it is also np-repulsing. As a consequence, our main Theorem 4 applies when both the logistics automaton and the constraint automaton are confluent. To prove this, we define the notion of confluence based on Milner's work on CCS [14] in which confluence is defined for labeled transition systems. To this end, we first define (following the definition in Reference [14]) for two activity sequences $\underline{b}$ and $\underline{c}$, the excess of $\underline{b}$ over $\underline{c}$, which is written as $\underline{b} / \underline{c}$. We obtain $\underline{b} / \underline{c}$ by working through $\underline{b}$ from left to right deleting any activity that occurs in $\underline{c}$, taking into account the multiplicity of occurrence. $\underline{b}$ as:

Definition 14 (Excess). Let $\underline{b}, \underline{c} \in \mathcal{A} c t^{*}$ be activity sequences. Then $\underline{b} / \underline{c}$ is defined recursively upon

$$
\begin{aligned}
\epsilon / \underline{c} & =\epsilon \\
(a \underline{b}) / \underline{c} & = \begin{cases}a(\underline{b} / \bar{c}) & \text { if } a \text { does not occur in } \underline{c} . \\
\underline{b} /(\underline{c} / a) & \text { if } a \text { occurs in } \underline{c}\end{cases}
\end{aligned}
$$

This excess operator has a number of important properties.

Lemma 15. Let $\underline{b}, \underline{c} \in \mathcal{A} c t^{*}$ be activity sequences and let $a \in \mathcal{A} c t$ be an activity. Then

(1) $\#_{a}(\underline{b} / \underline{c})=\max \left(\#_{a}(\underline{b})-\#_{a}(\underline{c}), 0\right)$, where $\#_{a}(\underline{b})$ denotes the number of occurrences of $a$ in $\underline{b}$;

(2) $\underline{b} \sim p \underline{c}$ if and only if $\underline{b} / \underline{c}=\epsilon$ and $\underline{c} / \underline{b}=\epsilon$;

(3) $\underline{b} / \underline{c}=\underline{b}$ if $b$ and c have no activities in common;

(4) $(\underline{b} / \underline{c}) /(\underline{c} / \underline{b})=\underline{b} / \underline{c}$.

We will now define the notion of confluence on logistic and constraint automata.

Definition 15 (Confluence). Let $L C=\left\langle\mathcal{S}, \mathcal{A} c t, \dot{\rightarrow}, \mathcal{S}_{0}\right\rangle$ be either a logistics automaton or a constraint. Then $L C$ is called confluent if either $\mathcal{S}=\emptyset$ or $\mathcal{S}=\left\{s_{0}\right\}$ and the following condition holds: 

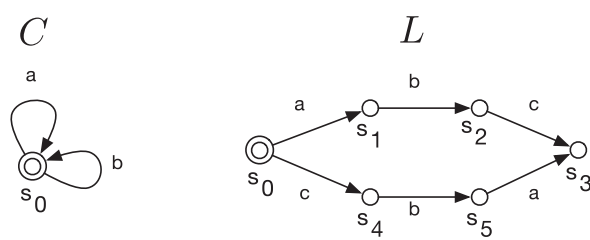

Fig. 10. A confluent constraint is not necessarily np-repulsing $(C)$ and an np-repulsing, $\mathrm{p}$ attracting logistics automaton is not necessarily confluent $(L)$.

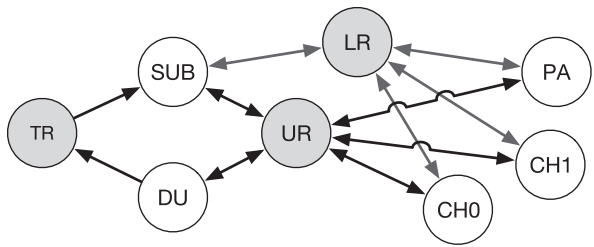

Fig. 11. Resources and possible product routes of the wafer handling controller.

- For all $s, s_{1}, s_{2} \in \mathcal{S}$ and $\underline{b}, \underline{c} \in \mathcal{A} c t^{*}$, if $s \stackrel{\underline{b}}{\rightarrow} s_{1}$ and $s \stackrel{\stackrel{c}{\rightarrow}}{\rightarrow} s_{2}$, then for some $s^{\prime} \in \mathcal{S}, s_{1} \stackrel{\underline{c} / \underline{b}}{\longrightarrow} s^{\prime}$ and $s_{2} \stackrel{\underline{b} / \underline{c}}{\longrightarrow} s^{\prime}$.

Every confluent logistics automaton or constraint is p-attracting, which is claimed by the following lemma:

Lemma 16. Let $L C=\left\langle\mathcal{S}, \mathcal{A c t}, \dot{\rightarrow}, \mathcal{S}_{0}\right\rangle$ be a confluent logistics automaton or constraint. Then $L C$ is p-attracting.

With respect to np-repulsiveness a similar relation holds, but only for logistics automata. A confluent constraint is not necessarily np-repulsing, which is shown in the following example:

Example 11. Consider constraint $C$ depicted in Figure 10. $C$ is confluent and p-attracting (consistent with Lemma 16). $C$ is not np-repulsing however, since the nonpermuting sequences $a$ and $a b$ both lead to state $s_{0}$.

A confluent logistics automaton, however, is always np-repulsive. This also holds for constraints that are non-recursive. This is posed in the following lemma:

Lemma 17. Let $C=\left\langle\mathcal{S}, \mathcal{A} c t, \dot{\rightarrow}, \mathcal{S}_{0}\right\rangle$ be a confluent logistics automaton or non-recursive constraint. Then $C$ is np-repulsing.

From Lemmas 16 and 17, we thus know that every confluent logistics automaton and nonrecursive constraint is both np-repulsing and p-attracting. The opposite is not true, which is demonstrated in the following example:

Example 12. Consider automaton $L$ depicted in Figure 10. $L$ is both np-repulsing and pattracting. But for instance, since $s_{1}$ cannot make a transition with label $c, L$ is not confluent.

For our main reduction Theorem 4 to apply, the logistics automaton should be np-repulsing and the constraint should be p-attracting. From Lemmas 16 and 17, we thus know this holds in case of a confluent automaton and constraint.

\section{EXPLOITING THE ALGEBRA}

We defined equivalence and inclusion relations on logistics automata and their languages and proved that these relations are substitutive under all operators. In addition, we proved commutativity, associativity, and distributivity properties and defined the notions of np-repulsiveness and p-attractiveness. With this, we defined an algebra on logistics automata. This algebra allows us to compare logistics specifications in a modular (algebraic) way by systematically relating their languages, their state-space and optimization-space sizes, and their solutions to the BMO problem. In addition, it allows the exploitation of over-specification by the systematic introduction of 
constraints to solve the BMO problem (for the constrained system) or BMO bounds (for the unconstrained system). Hence, the algebra facilitates a specification style and approach to keep the makespan optimization problem in check. This is illustrated by the following examples:

Example 13. Assume that we want to compare specifications $\left(\left(\left(L_{1} \odot L_{2}\right)\left\lceil C_{1}\right) \odot L_{3}\right) \uparrow C_{2}\right.$ and $\left(\left(L_{1} \odot L_{2}\right)\left\lceil C_{1}\right) \odot L_{3}\right.$ where $C_{2}$ is a constraint on $L_{1}, L_{2}$, and $L_{3}$ and $L_{1}, L_{2}$, and $L_{3}$ are np-repulsing automata and $C_{2}$ is p-attracting. We can use our algebra to "massage" specification $\left(\left(\left(L_{1} \odot L_{2}\right) \uparrow\right.\right.$ $\left.\left.C_{1}\right) \odot L_{3}\right) \uparrow C_{2}$ as follows:

$\left(\left(\left(L_{1} \odot L_{2}\right)\left\lceil C_{1}\right) \odot L_{3}\right)\left\lceil C_{2}\right.\right.$

$\approx\{$ Distributivity of $\uparrow($ Theorem 3.4$)\}$

$\left(\left(L_{1} \odot L_{2}\right)\left\lceil C_{1}\left\lceil C_{2}\right) \odot\left(L_{3}\left\lceil C_{2}\right)\right.\right.\right.$

$\approx$ Commutativity of $\uparrow$ (Theorem 3.3), substitutivity of $\approx$ under $\odot$ (Theorem 3.6)\}

$\left(\left(L_{1} \odot L_{2}\right)\left\lceil C_{2}\left\lceil C_{1}\right) \odot\left(L_{3}\left\lceil C_{2}\right)\right.\right.\right.$

$\approx$ \{Distributivity of $\uparrow$ (Theorem 3.4), substitutivity of $\approx$ under $\uparrow$ (Theorem 3.10)

and substitutivity of $\approx$ under $\odot$ (Theorem 3.6$)\}$

$\left(\left(L_{1}\left\lceil C_{2} \odot L_{2}\left\lceil C_{2}\right) \uparrow C_{1}\right) \odot\left(L_{3}\left\lceil C_{2}\right)\right.\right.\right.$

$\sqsubseteq\left\{L_{1} \uparrow C_{2} \sqsubseteq L_{1}, L_{2} \uparrow C_{2} \sqsubseteq L_{2}\right.$ and $L_{3} \uparrow C_{2} \sqsubseteq L_{3}$ by Theorem 4 ,

substitutivity of $\sqsubseteq$ under $\uparrow($ Theorem 3.9) and $\odot$ (Theorem 3.5) and

commutativity of $\odot$ (Theorem 3.1)\}

$\left(\left(L_{1} \odot L_{2}\right) \uparrow C_{1}\right) \odot L_{3}$

Hence, by Theorem $\left.3.23 \mathcal{L}\left(\left(\left(L_{1} \odot L_{2}\right) \uparrow C_{1}\right) \odot L_{3}\right) \uparrow C_{2}\right) \subseteq \mathcal{L}\left(\left(\left(L_{1} \odot L_{2}\right) \uparrow C_{1}\right) \odot L_{3}\right)$. Therefore, a solution to the BMO problem of $\left(\left(\left(L_{1} \odot L_{2}\right)\left\lceil C_{1}\right) \odot L_{3}\right)\left\lceil C_{2}\right.\right.$ is a suboptimal solution to the BMO problem of $\left(\left(L_{1} \odot L_{2}\right)\left\lceil C_{1}\right) \odot L_{3}\right.$ establishing a makespan bound thereof (Lemma 5). Further, by Lemma 10 the state-space of $\left(\left(\left(L_{1} \odot L_{2}\right)\left\lceil C_{1}\right) \odot L_{3}\right)\left\lceil C_{2}\right.\right.$ is at most as large as that of $\left(\left(L_{1} \odot L_{2}\right) \uparrow\right.$ $\left.C_{1}\right) \odot L_{3}$ and by Theorem 3.13 and Lemma 10 the optimization-space of $\left(\left(\left(L_{1} \odot L_{2}\right)\left\lceil C_{1}\right) \odot L_{3}\right)\left\lceil C_{2}\right.\right.$ is at most as large as that of $\left(\left(L_{1} \odot L_{2}\right)\left\lceil C_{1}\right) \odot L_{3}\right.$. Since the optimization-space of the constrained specification $\left(\left(\left(L_{1} \odot L_{2}\right)\left\lceil C_{1}\right) \odot L_{3}\right) \uparrow C_{2}\right.$ is at most as large as that of the unconstrained specification $\left(\left(L_{1} \odot L_{2}\right)\left\lceil C_{1}\right) \odot L_{3}\right.$, the effort to compute a solution is either smaller or equal to that of computing a solution of the unconstrained specification $\left(\left(L_{1} \odot L_{2}\right)\left\lceil C_{1}\right) \odot L_{3}\right.$. Therefore, the suboptimal solution and makespan bound of the BMO problem of $\left(\left(L_{1} \odot L_{2}\right)\left\lceil C_{1}\right) \odot L_{3}\right.$ is therefore easier to compute than the optimal solution and makespan thereof.

Example 14. Now assume that we want to reduce the state-space and optimization-space of $\left(L_{1} \uparrow C_{1} \odot L_{2}\right) \uparrow C_{2}$ where $L_{2}$ is an np-repulsing automaton. We can do so by applying some pattracting constraint $C_{3}$ to $L_{2}$. Then, by Theorem 4, we know that $L_{2}\left\lceil C_{3} \sqsubseteq L_{2}\right.$. Subsequently by applying substitutivity of $\sqsubseteq$ under $\odot$ (Theorem 3.5), we have $\left(L_{1}\left\lceil C_{1} \odot L_{2}\left\lceil C_{3}\right) \sqsubseteq\left(L_{1}\left\lceil C_{1} \odot L_{2}\right)\right.\right.\right.$. But then by applying substitutivity of $\sqsubseteq$ under $\left\lceil\right.$ (Theorem 3.9), we have $\left(L_{1}\left\lceil C_{1} \odot L_{2}\left\lceil C_{3}\right) \uparrow C_{2} \sqsubseteq\right.\right.$ $\left(L_{1}\left\lceil C_{1} \odot L_{2}\right)\left\lceil C_{2}\right.\right.$. Similar to Example 13, we can now compute the BMO solution to $\left(L_{1}\left\lceil C_{1} \odot\right.\right.$ $L_{2}\left\lceil C_{3}\right) \uparrow C_{2}$. This gives a suboptimal solution to the BMO problem of $\left(L_{1} \uparrow C_{1} \odot L_{2}\right) \uparrow C_{2}$, but it can be computed more efficiently than the optimal solution.

Using our algebra of logistics automata, we can define a systematic approach for the batchoriented specification and optimization of the logistics of flexible manufacturing systems as follows: 
(1) For each product in a batch, we write its product flow as an np-repulsing logistics automaton. Note that this can always be done, since any logistics automaton can be written as a language-equivalent automaton that is np-repulsing. For example, any logistics automaton $L$ can be written in its tree form Tree $(L)$, which has the same language and is np-repulsing. In some cases the product automata are actually confluent and also nprepulsing (Lemma 17).

(2) The automaton describing a batch is then obtained via the composition of all individual product automata. By Lemma 12, we know that the resulting automaton is also nprepulsing.

(3) Each system constraint should preferably be written as a p-attracting (or even confluent) constraint automaton. This is important, since by Theorem 4 and Lemma 10 the constraining of an np-repulsing automaton with a p-attracting constraint automaton leads to a reduction of the state-space and optimization-space of the logistics automaton. If a system constraint cannot be written (or conveniently written) as a p-attracting automaton step 4 (applying heuristics) is still applicable. This is because the constraining of a logistics automaton with any constraint preserves its np-repulsiveness (Lemma 13).

(4) In some cases the optimization-space is still too large to efficiently compute a solution to the $\mathrm{BMO}$ problem. In these cases, we can introduce additional (non-essential) constraints to the specification. These should be written as p-attracting (or even confluent) constraint automata such that the optimization-space is effectively pruned, following Theorem 4 and Lemma 10.

Using this systematic approach to the specification of batch-oriented logistics one can keep the makespan optimization problem in check. Further, it allows the exploitation of over-specification by the systematic introduction of additional requirements to solve the BMO problem (for the overconstrained system) or to find BMO makespan bounds (for the initial system specification). Notice that the technique does not allow us to make a quantitative statement about the quality of the solution obtained, if the unconstrained optimization-space can not be computed.

\section{CASE STUDY}

This section presents a case study in which we apply our framework to specify a wafer handling controller and optimize its makespan. The wafer handling controller is one of the controllers of an ASML TWINSCAN ${ }^{\mathrm{TM}}$ lithography scanner. These are manufacturing systems responsible for the photo-lithography step in the semiconductor manufacturing process where circuit patterns are exposed onto silicon wafers. Before this expose operation can occur, wafers must be correctly conditioned (for temperature and orientation) and measured to detect defects on its surface. In the case of the TWINSCAN ${ }^{\mathrm{TM}}$, the measure and expose operations are decoupled, which allows the system to increase productivity by executing them in parallel. To achieve this, the scanner contains two chucks that can change locations to perform either the measure or the expose operation.

We consider the input of the system to be a batch of 25 wafers, which is the typical number of wafers stored in a Front Opening Unified Pod (FOUP) [10]. In the next section, we describe the system in terms of its resources, activities, logistics, and system requirements. We omit any specification in terms of locations, layout, actions, and execution times for confidentiality reasons.

\subsection{Resources and Activities}

For wafer handling eight resources are utilized, which are depicted in Figure 11 by annotated circles. Circles with a white background represent the production resources SUB, DU, PA, CH0, and $\mathrm{CH} 1$, while darkened circles represent transport resource TR, LR, and UR. There are two robots that 
Table 1. Set of Activities of the Wafer Handling Controller

\begin{tabular}{lllll}
\hline (a) Track_2_SUB & (b) SUB_Conditioning & (c) SUB_2_UR & (d) UR_2_PA & (e) PA_PreAlign \\
(f) PA_2_LR & (g) LR_2_CH0 & (h) CH0_Measure & (i) CH0_Expose & (j) CH0_2_UR \\
(k) LR_2_CH1 & (1) CH1_Measure & (m) CH1_Expose & (n) CH1_2_UR & (o) UR_2_DU \\
(p) CH0_M_2_E & (q) CH1_M_2_E & (r) CH0_E_2_M & (s) CH1_E_2_M & \\
\hline
\end{tabular}

are used to transport wafers between the different processing units, the Load Robot (LR) and the Unload Robot (UR). The Storage Unit (SUB) ensures that each wafer is conditioned to a predefined temperature. The Pre-Aligner (PA) accurately aligns a wafer with respect to a reference position. The Discharge Unite (DU) is an output buffer. The Track (TR) is a system external to the system responsible for the input and output to and from the scanner. Finally, we have two chucks $(\mathrm{CH} 0$ and $\mathrm{CH} 1$ ) that are able to perform the scanner operations measure and expose.

Table 1 lists all the activities necessary to capture the good-weather manufacturing of a batch of wafers. In this context good-weather means that we do not account for error or test scenarios. Therefore, many of the possible movement operations between resources, depicted by the directional arrows in Figure 11, are not utilized. To avoid clutter in the figures and text, we will refer to these activities using bold-case letters as indicated in Table 1 (e.g., activity Track_2_SUB is referred by letter a). To denote an activity that is performed on behalf of wafer $i$, we attach a corresponding subscript to the activity name. For example, activity a_3 represents activity Track_2_SUB on behalf of wafer 3 . Activity a captures the input operation during which a wafer is loaded into the scanner by the TR resource. Similarly, activity o captures the output of an exposed wafer from the system by placing it on the DU resource. Activities $\mathbf{b}$ and $\mathbf{e}$ capture the pre-processing operations concerning conditioning and pre-aligning. Activity $\mathbf{b}$ conditions the wafer temperature for an accurate exposure and activity e aligns the wafer to minimize the overlay error of multiple exposures. Activities $\mathbf{l}$ and $\mathbf{h}$ capture the measure operations on the $\mathrm{CH} 0$ and $\mathrm{CH} 1$ resources, respectively, and activities $\mathbf{m}$ and $\mathbf{i}$ capture the expose operations on the $\mathrm{CH} 0$ and $\mathrm{CH} 1$ resources, respectively. Resources $\mathrm{CH} 0$ and $\mathrm{CH} 1$ need to be in specific locations to perform the measure and expose operations. Activities p, q, r, and s capture the movement of resources $\mathrm{CH} 0$ and $\mathrm{CH} 1$ from and to the measure and expose locations. For example activity CH0_M_2_E (p) captures the moving of resource $\mathrm{CH} 0$ from the measure location (M) to the expose location (E). Activities $\mathbf{c}, \mathbf{d}, \mathbf{f}$, $\mathbf{j}, \mathbf{g}, \mathbf{k}, \mathbf{n}$, and $\mathbf{o}$ capture the movement of wafers across the system using resources LR and UR. These are always described by an initial and destination resource. For example, activity PA_2_LR (f) captures the movement of a wafer from the PA resource to the LR resource.

\subsection{Wafer Logistics}

For every wafer, we define its life-cycle (wafer product flow). Figure 12 depicts logistics automaton $L_{\mathrm{LC}}$, which captures the life-cycle of wafers $i$ in a batch of wafers. This life-cycle starts with the input of a wafer into the scanner by placing it on the SUB resource. This is done by the TR resource (activity a_i). Once on the SUB resource, the wafer is conditioned to a pre-defined range of temperatures (activity $\mathbf{b} \_$i). After conditioning, the wafer is moved from the SUB resource to the PA resource using the UR resource as an intermediary (activities $\mathbf{c}_{-} i$ and $\left.\mathbf{d} \_i\right)$ ). The PA resource accurately aligns the wafer to a reference (activity $\mathbf{e}$ _i). Once aligned, the wafer is picked by the LR resource from the PA (activity $\mathbf{f}$ i). At this point the controller has a choice to either load the wafer on chuck resource $\mathrm{CH} 0$ or on chuck resource $\mathrm{CH} 1$, (activity $\mathbf{g} \_\mathrm{i}$ or activity $\mathbf{k}$ _i, respectively). This is visible in automata $L_{\mathrm{LC}_{i}}$ by the branching after activity $\mathbf{f}$. i. Assume that the controller picks the $\mathrm{CH} 0$ resource (upper branch). In this case, the wafer is measured and then exposed using resource 


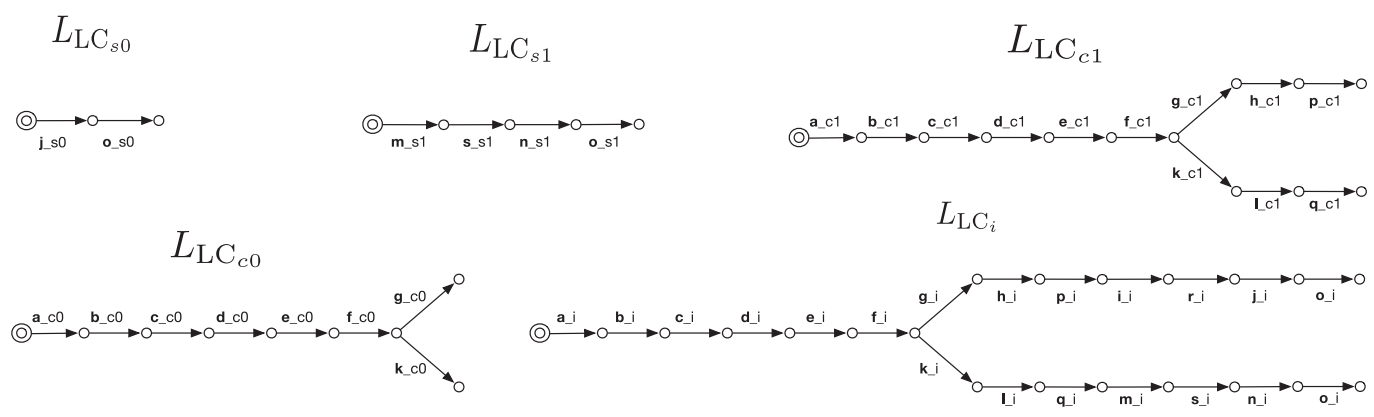

Fig. 12. Logistics automaton $L_{\mathrm{LC}_{i}}$ capturing the life-cycle for a wafer $i$ of a batch of wafers and logistics automata $L_{\mathrm{LC}_{s 0}}, L_{\mathrm{LC}_{s 1}}, L_{\mathrm{LC}_{c 1}}$, and $L_{\mathrm{LC}}$ capturing the life-cycle of dummy wafers $\mathrm{s} 0, \mathrm{~s} 1, \mathrm{c} 1$, and $\mathrm{c} 0$, respectively.

$\mathrm{CH}$. After measuring (activity $\mathbf{h} \_\mathrm{i}$ ), resource $\mathrm{CH} 0$ moves from the measure location to the expose location (activity $\mathbf{p} \_$i). Once in the expose location the wafer is exposed with a certain circuit pattern (activity i_i). Then resource $\mathrm{CH} 0$ moves from the expose location to the measure location (activity $\mathbf{r}_{-} \mathrm{i}$ ). Once returned at the measure location, the wafer is moved from the $\mathrm{CH} 0$ resource to the UR resource and finally placed on the DU resource to be outputted by the TR resource (activities $\mathbf{j}_{-} \mathrm{i}$ and $\mathbf{o} \_\mathrm{i}$, respectively). In case the controller picks the $\mathrm{CH} 1$ the product flow remains similar, however, the activities for the $\mathrm{CH} 1$ resource would be executed $\mathbf{k} \_\mathrm{i}, \mathbf{l} \_\mathrm{i}, \mathbf{q} \_\mathrm{i}, \mathbf{m} \_\mathrm{i}, \mathbf{s} \_\mathrm{i}$, and n_i.

Whenever the system is not in production, dummy wafers are loaded on the chuck resources for safety reasons. For this case-study, we take this into account by modeling four dummy wafers $\mathrm{s} 0$, $\mathrm{s} 1, \mathrm{c} 0$, and $\mathrm{c} 1$. Dummy wafers s0 and s1 represent the dummy wafers already in the system before the processing of a FOUP, while $\mathrm{c} 0$ and $\mathrm{c} 1$ represent the dummy wafers that should be loaded once the FOUP is processed. We assume that $\mathrm{CHO}$ starts at the measure location and that $\mathrm{CH} 1$ starts at the expose location. This is enforced in the life-cycle of dummy wafers $\mathrm{s} 0$ and s1 captured by logistics automata $L_{\mathrm{LC}_{s 0}}$ and $L_{\mathrm{LC}_{s 1}}$, depicted in Figure 12. The life-cycle of s0 starts with the unloading of the dummy wafer from the $\mathrm{CH} 0$ resource to the UR resource (activity $\mathbf{j}_{-} \mathrm{s} 0$ ) and ends with its placement on the output buffer DU (activity o_s0). The life-cycle of s1 starts with the expose activity of dummy wafer $\mathrm{s} 1$ (activity $\mathbf{m} \_\mathrm{s} 1$ ) followed by the movement of the $\mathrm{CH} 1$ resource to the measure location (activity s_s1), its unloading to the UR resource (activity $\mathbf{n} \_s 1$ ), and finally its placement on the output buffer DU (activity o_s1). The life-cycle of dummy wafers c0 and c1 follows the same flow as the life-cycle of a production wafer but ends in different final states. These are captured by logistics automata $L_{\mathrm{LC}}$ and $L_{\mathrm{LC}}$, depicted in Figure 12 . The life-cycle of $\mathrm{c} 0$ ends once the dummy wafer is loaded onto either the $\mathrm{CH} 0$ resource or the $\mathrm{CH} 1$ resource (activity g_c0 or $\left.\mathbf{k} \_c 0\right)$. The life-cycle of $\mathrm{c} 1$ ends once the dummy wafer is moved to the expose location placed either on the $\mathrm{CH} 0$ resource and $\mathrm{CH} 1$ resource (activity p_c1 or q_c1).

The batch specification of a batch of 25 wafers is given by the following logistics expression: $L_{\mathrm{LC}_{s 0}} \odot L_{\mathrm{LC}_{s 1}} \odot\left(L_{\mathrm{LC}_{1}} \odot L_{\mathrm{LC}_{2}} \odot \cdots \odot L_{\mathrm{LC}_{25}}\right) \odot L_{\mathrm{LC}_{c 1}} \odot L_{\mathrm{LC}_{c 0}}$. For brevity, we will refer to this composite automaton as $L_{\mathrm{LC}}$. Note that each of the life-cylce automata in Figure 12 is np-repulsing and that by Lemma 12 the composition $L_{\mathrm{LC}}$ is therefore also np-repulsing.

\subsection{System Requirements}

On top of the product flow there are certain functional requirements on the manufacturing of a batch of wafers. These are listed and explained below: 


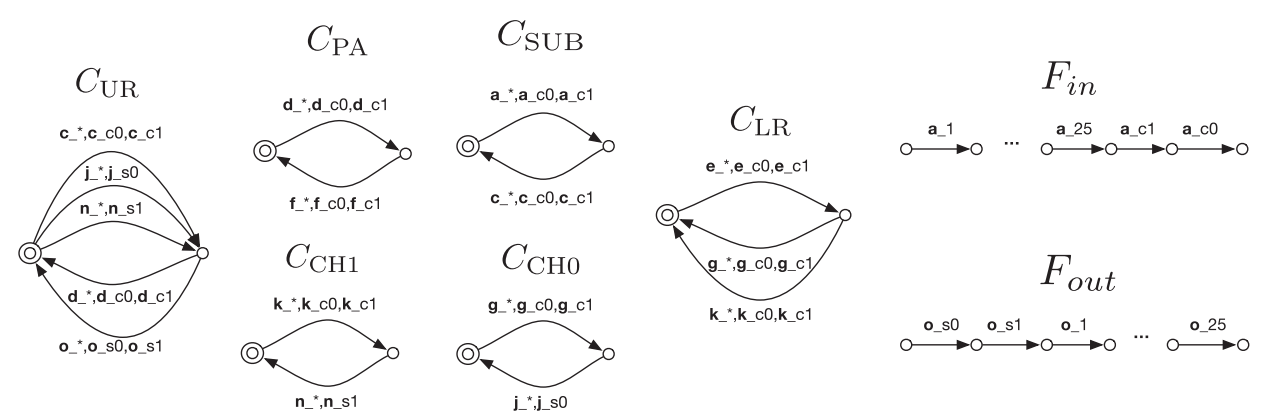

Fig. 13. Constraint automata $C_{\mathrm{SUB}}, C_{\mathrm{UR}}, C_{\mathrm{LR}}, C_{\mathrm{PA}}, C_{\mathrm{CH} 0}$, and $C_{\mathrm{CH} 1}$ capturing capacity requirements for the wafer handling resources and constraint automata $F_{\text {in }}$ and $F_{\text {out }}$ capturing the FIFO input and output ordering.

(1) Products shall enter and leave the system in a First-In-First-Out (FIFO) order.

(2) There shall only be one product at a time in each resources (unary capacity).

(3) Wafers shall not collide (i.e., products shall not be be placed on a occupied resource).

(4) Chucks must swap positions in between every measure and expose activity (Swap).

(5) Chucks must be loaded and unloaded at the measure location.

Requirement 1 is captured by constraint automata $F_{\text {in }}$ and $F_{\text {out }}$, depicted in Figure 13 . This is done by ordering input and output activities a and $\mathbf{o}$ for all wafers in the FOUP and dummy wafers s0, $\mathrm{s} 1, \mathrm{c} 0$, and $\mathrm{c} 1$. Requirements 2 and 3 are satisfied by enforcing that activities that place a wafer on a resource can only occur after a corresponding activity that picks a wafer from that resource and vice versa. This is captured by p-attracting constraint automata $C_{\mathrm{SUB}}, C_{\mathrm{UR}}, C_{\mathrm{LR}}, C_{\mathrm{PA}}, C_{\mathrm{CH} 0}$, and $C_{\mathrm{CH} 1}$, depicted in Figure 13. For example, consider automata $C_{\mathrm{SUB}}$. The initial state represents the resource to be empty and the other state represents the resource to be occupied. To avoid cluttering the automata figures, we write act_* to represent 25 different transitions with labels act_1, . . act_25 corresponding to the 25 production wafers in a FOUP (where act denotes the name of an activity). If the resource is empty, activities $\mathbf{a}_{-} *, \mathbf{a}_{-} \mathrm{c} 1$, and $\mathbf{a}_{-} \mathrm{c} 0$ are enabled, since these imply the placing of wafer on the SUB. If an activity $\mathbf{a}_{-} *$, $\mathbf{a}_{-} \mathrm{c} 1$, or $\mathbf{a} \_\mathrm{c} 0$ is executed, then it is enforced that another activity $\mathbf{a}_{-} *, \mathbf{a} \_\mathrm{c} 1$, or $\mathbf{a} \_\mathrm{c} 0$ can only occur if an activity $\mathbf{c}_{-} *, \mathbf{c} \_\mathrm{c} 1$, or $\mathbf{c}_{-} \mathrm{c} 0$ occurs in between.

Requirements 4 and 5 are partially enforced by the logistics product flow $L_{\mathrm{LC}_{i}}$ and partially by p-attracting constraint automata $C_{\text {Swap }}, C_{\text {UnloadLoad }_{0}}$, and $C_{\text {UnloadLoad }_{1}}$, depicted in Figure 14. Constraint automaton $C_{S w a p}$ enforces that after every execution of the measure and expose activities, chuck resources $\mathrm{CH} 0$ and $\mathrm{CH} 1$ swap positions. The initial state of $C_{\text {Swap }}$ considers two initial situations: (1) $\mathrm{CH} 0$ is at the measure location and $\mathrm{CH} 1$ is at the expose location or (2) $\mathrm{CH} 1$ is at the measure location and $\mathrm{CH} 0$ is at the expose location. Situation (1) is captured by the lower branches and (2) by the top branches. After executing the corresponding measure and expose operation the chuck resources $\mathrm{CH} 1$ and $\mathrm{CH} 0$ swap locations. In situation (1) $\mathrm{CH} 0$ moves from measure to expose (activities $\mathbf{p}_{-}{ }^{*}$ and $\mathbf{p}_{-} \mathrm{c} 1$ ) and $\mathrm{CH} 1$ from expose to measure (activities $\mathbf{s}_{-}^{*}$ and $\mathbf{s}_{-} \mathrm{s} 1$ ) and in (2) $\mathrm{CH} 1$ moves from measure to expose (activities $\mathbf{q}_{-}{ }^{*}$ or $\mathbf{q}_{-} \mathrm{c} 1$ ) and $\mathrm{CH} 0$ from expose to measure (activities $\mathbf{r}_{-}^{*}$ ). This flow is then repeated for each of the initial situations captured by cases (1) and (2). P-attracting constraint automata $C_{\text {UnloadLoad }_{0}}$ and $C_{\text {UnloadLoad }_{1}}$ enforce that the loading and unloading of a chuck to and from resources $\mathrm{CH} 0$ and $\mathrm{CH} 1$, respectively, only occurs if the respective chuck resource is at the measure location. In the case of resource $\mathrm{CH} 0$ (captured by $C_{\text {UnloadLoad }_{0}}$ ), its starting position is at the measure location and therefore the initial state enables both the loading 

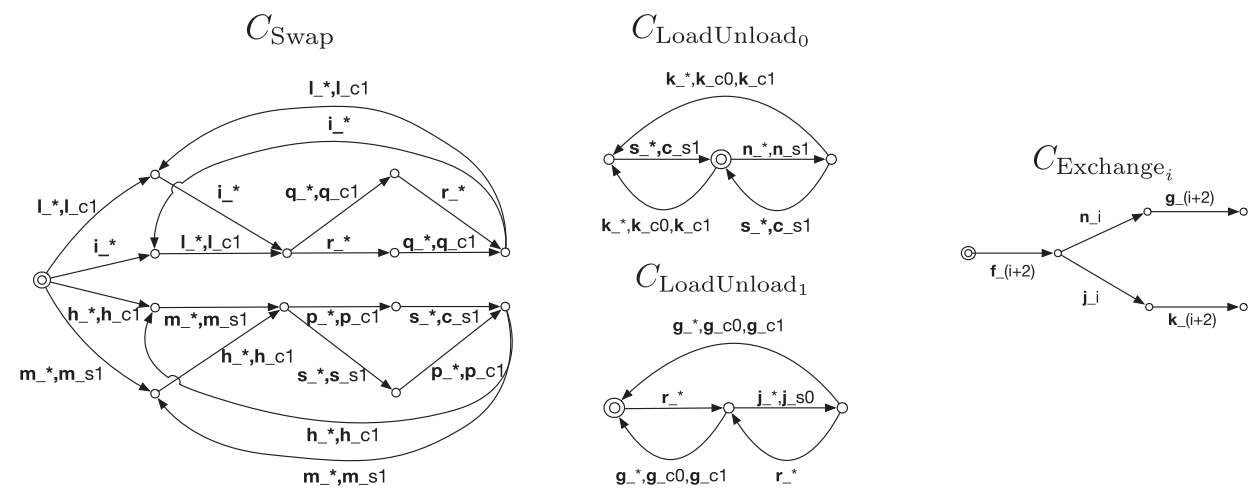

Fig. 14. Constraint $C_{\text {Swap }}$ capturing the exchange of chuck positions after measure and expose operations; constraint $C_{\text {UnloadLoad }_{0}}$ and $C_{\text {UnloadLoad }_{1}}$ enforce that the load/unload of wafers occurs at the measure location and constraint $C_{\text {Exchange }}$ captures a specific order of the exchange of wafers from and to $\mathrm{CH} 0$ and $\mathrm{CH} 1$.

Table 2. Size of the State-space and Optimization-space, and Computed Makespan for a FOUP (25 Wafers and 4 Dummy Wafers)

\begin{tabular}{ccccccc}
\hline & \multicolumn{2}{c}{ State-Space } & & \multicolumn{2}{c}{ Optimization-Space } & \\
\cline { 2 - 3 } Model & N. States & N. Transitions & & N. States & N. Transitions & Makespan (s) \\
\hline Logistics & - & - & - & - & - & - \\
\hline +Capacity & - & - & - & - & - \\
+FIFO & 65,823 & 216,611 & & - & - \\
+Swap & 13,630 & 39,738 & 772,806 & $2,200,049$ & 331.7 \\
\hline +Exchange & 9,778 & 28,225 & 264,854 & 737,499 & 331.7 \\
\hline
\end{tabular}

(activities $\mathbf{n}_{-}{ }^{*}$ or $\mathbf{n} \_$s1) and the unloading (activities $\mathbf{k}_{-}{ }^{*}, \mathbf{k}_{-} \mathrm{c} 0$, or $\mathbf{k}_{-} \mathrm{c} 1$ ) of a wafer. If the wafer is unloaded the automaton moves to a state where it still enables the loading of another wafer. Once a wafer is loaded onto the resource, then the automata moves to a state where it first requires that the chuck resource moves again to the measure location before allowing it to load or unload a wafer again. The flow is similar for $\mathrm{CH} 1$ with the difference that the $\mathrm{CH} 1$ resource starts at the expose location.

The specification of a batch of 25 wafers is now obtained by constraining logistics automaton $L_{\mathrm{LC}}$ with all of the (p-attracting) constraints described above: $L_{\mathrm{LC}}\left\lceil C_{\mathrm{COND}}\left\lceil C_{\mathrm{UR}}\left\lceil C_{\mathrm{LR}}\left\lceil C_{\mathrm{PA}}\left\lceil C_{\mathrm{CH} 0} \uparrow\right.\right.\right.\right.\right.$ $C_{\mathrm{CH} 1}\left\lceil F_{\text {in }}\left\lceil F_{\text {out }} \uparrow C_{\text {Swap }}\left\lceil C_{\text {UnloadLoad }_{1}}\left\lceil C_{\text {UnloadLoad }_{1}}\right.\right.\right.\right.$.

\subsection{Wafer Logistics Optimization}

Now that we have introduced the wafer handling controller, its logistics requirements, and system constraints, we compute the optimization-space and the solution to the BMO for a batch of 25 wafers (including the 4 dummy wafers $s 0, s 1, c 0$, and $c 1$ ). The values presented in this section are computed in a system with an Intel i7 920@2.67 Ghz with 8 cores and 32 GB of RAM memory. We use the CIF tool [18] to compute the state-spaces of logistics automata and the algorithms of References $[6,19]$ to compute the corresponding optimization-spaces and optimal activity sequences.

Table 2 shows the results in terms of the number of states (N. States) and transitions (N. Transitions) of the state-space and optimization-space as well as the obtained minimal makespan (Makespan) for the processing of a FOUP by the wafer handler. In case we are not able to 
compute the state-spaces this is noted with a (-) symbol. The values for the state-space and optimization-space sizes of $L_{\mathrm{LC}}$ are shown in the first row (Logistics) of Table 2. Each additional row of the table represents the cumulative addition of another requirement (indicated by the + symbol). Where + Capacity refers to automaton $\left(L_{\mathrm{LC}}\right)\left\lceil C_{\mathrm{COND}}\left\lceil C_{\mathrm{UR}}\left\lceil C_{\mathrm{LR}}\left\lceil C_{\mathrm{PA}} \uparrow C_{\mathrm{CH} 0} \uparrow\right.\right.\right.\right.$ $C_{\mathrm{CH} 1},+\mathrm{FIFO}$ to automaton $\left(L_{\mathrm{LC}} \uparrow C_{\mathrm{COND}} \uparrow C_{\mathrm{UR}} \uparrow C_{\mathrm{LR}} \uparrow C_{\mathrm{PA}} \uparrow C_{\mathrm{CH} 0} \uparrow C_{\mathrm{CH} 1}\right) \uparrow F_{\text {in }} \uparrow F_{\text {out }}$, and + Swap to automaton $\left(L_{\mathrm{LC}} \uparrow C_{\mathrm{COND}} \uparrow C_{\mathrm{UR}} \uparrow C_{\mathrm{LR}} \uparrow C_{\mathrm{PA}} \uparrow C_{\mathrm{CH} 0} \uparrow C_{\mathrm{CH} 1} \uparrow F_{\text {in }} \uparrow F_{\text {out }}\right) \uparrow C_{\mathrm{Swap}} \uparrow C_{\text {UnloadLoad }_{1}} \uparrow$ $C_{\text {UnloadLoad }_{1}}$. We observe that the cumulative constraining of the system with Capacity, FIFO and Swap requirements systematically reduces the size of the state-space and optimization-space until the point that the optimization-space can be explicitly constructed and a solution to the BMO problem can be computed, yielding a minimal makespan of 331.7 seconds. For the specifications that we are able to compute the logistics automaton, a solution to the BMO problem is computed within 2 minutes. Even though it is sufficient to apply all system constraints to compute the makespan optimal activity sequence, we demonstrate the effectiveness of the heuristics approach by further pruning the state-space and optimization-space. Since the Capacity, FIFO, and Swap requirements are p-attracting, we know that their application to $L_{\mathrm{LC}}$ resulted in an np-repulsing automaton. The optimization space can thus be pruned further by applying additional p-attracting automata. To illustrate this, we will use over-specification by subsequently formalizing a non-essential requirement.

Each wafer must eventually be placed on either the $\mathrm{CH} 0$ or $\mathrm{CH} 1$ resource of the expose stage for the measure and expose activities to take place. This is realized by a combination of activities $\mathbf{j} \_\mathrm{i}$ and $\mathbf{g} \_\mathrm{i}$ or $\mathbf{n} \_\mathrm{i}$ and $\mathbf{k} \_\mathrm{i}$. The process of loading and unloading a wafer to and from $\mathrm{CH} 0$ and $\mathrm{CH} 1$ always takes place from the measure location. Therefore, chucks $\mathrm{CH} 0$ and $\mathrm{CH} 1$ must always move first to the measure location before exchanging an exposed wafer for a new wafer. Further, to maximize productivity both chucks should be utilized in an effort to parallelize the measure and expose activities. For these reasons an exchange should always consider an exposed wafer $i$ and an non-exposed wafer $i+2$. Due to this, we figured that a proper heuristic is defined by pattracting constraint automaton $C_{\text {Exchange }_{i}}$ (depicted in Figure 14), which enforces a specific order between unload of wafer $i$ from either $\mathrm{CH} 0$ or $\mathrm{CH} 1$ and the load of wafer $i+2$ to the corresponding chuck resource. This constraint is instantiated for each wafer $i$ where $1 \leq i \leq 25$. For the last two dummy wafers this requirement is not enforced. The results are shown in Table 2 in the + Exchange row. Because we could already compute the optimal solution without over-specification, we can compare quantitatively the impact of the additional constraint: It reduces the optimization-space by more than $60 \%$. Note that the optimal result is preserved even though the system is further constrained with over-specification in this case.

\section{RELATED WORK}

To the best of our knowledge, this is the first work in which an algebraic framework is developed to systematically reason about state-space sizes. This work combines the ingredients from the modular constraint-oriented approach of the LOTOS framework [8] and of Supervisory Control Theory (SCT) [16] developed in Reference [19] and applied in Reference [12]. We build upon the general concepts of SCT, but refine this framework by allowing only non-recursive requirements and by explicitly distinguishing logistics automata from constraint automata.

To get insight in the impact of automata composition on the state-space sizes, we took inspiration from the Calculus of Communication Systems (CCS) [14], in particular from Milner's simulation relation $\prec$. This relation captures behavior but abstracts from all structural information of the automata. We strengthen this relation by adding structural information (in the form of an injectivity requirement) to allow qualitative reasoning about state-space sizes. In addition, it benefits from the effective proof technique of establishing simulation relations. The strengthening of pre-order 
$<$ makes $\sqsubseteq$ into a partial-order. As far as we have been able to verify, this is the first work in which the inclusion relation $\sqsubseteq$ (to compare transitions systems in both a behavioral and in a structural way) is established, together with its algebraic properties.

It is important to mention that for some instances of the BMO problem other heuristics-based and approximation techniques could be applicable. For example, by using heuristics from different job-shop [2] or vehicle routing [7] problems.

In addition to our approach, some of these solutions include deadlines and due dates [21-23] and other types of timing constraints $[3,4]$. However, they do not focus on expressing or guaranteeing the satisfaction functional requirements. Moreover, the application of such techniques and algorithms is quite dependent on the actual instance of the problem we might be trying to solve. In Reference [2] a survey of different solutions to diverse flexible job-shop formulation is carried out, considering systems with multiple operation assignments, multiple transport routing choices, resource sharing, setup times and other system requirements. We conjecture that our work can specify and optimize all of these variants, except those dealing with timing constraints (e.g., deadlines and due dates). Notice that a direct comparison of these optimization approaches to the design framework presented would not be a fair, since it would focus solely on the makespan computation and not on other features such as the specification methodology and the algebraic framework or guaranteeing the satisfaction of functional requirements.

\section{CONCLUSIONS}

In this article, we extended the framework of References $[6,19]$ by introducing an algebra on logistics automata. This algebra allows us to compare logistics specifications in a modular (algebraic) way, by relating their languages, their state-space and optimization-space sizes and their solutions to the BMO problem. To this end, we defined equivalence and inclusion relations on logistics automata and their languages and established important algebraic properties for the composition $(\odot)$, constraining $(\uparrow)$, MaxPlus, and Tree operators. We showed that our inclusion relation is a partialorder relation and that if a logistics automaton is included in another, the state-space of the former never exceeds that of the latter. Further, we showed that a logistics automaton is always included in its corresponding $(\max ,+)$ automaton.

We also established sufficient conditions to ensure that the constraining of a logistics automaton leads to the pruning of its state-space. To this end, we defined the notions of np-repulsiveness and p-attractiveness and showed: (1) that np-repulsiveness and p-attractiveness are preserved under the composition operator; (2) that np-repulsiveness is preserved by general constraining; and (3) that the constraining of an np-repulsing logistics automaton with a p-attracting constraint automaton effectively reduces the size of the state-space and optimization-space of the logistics automaton. Further, we related the notions of np-repulsiveness and p-attractiveness to the notion of confluence and showed that: (1) a confluent logistics automaton is both np-repulsing and p-attracting, but not vice versa; (2) a confluent constraint automaton is p-attracting, but not necessarily np-repulsing.

Using the algebra of logistics automata and its properties, we defined a method for the batchoriented specification of the logistics of flexible manufacturing systems. In essence, logistics requirements should be written as np-repulsing logistics automata and constraints as p-attracting constraint automata. Satisfying these conditions ensures that constraining the logistics automaton effectively reduces the size of its state-space and optimization-space. Furthermore, it also allows the exploitation of over-specification by the systematic introduction of additional requirements to solve the BMO problem (for the over-constrained system) or to find BMO makespan bounds (for the initial non over-constrained system specification). This method facilitates a specification style and optimization approach to keep the makespan optimization problem in check. 
We demonstrated the applicability of the algebra and of the specification method by solving the BMO problem of an industrial case study of a wafer handling controller as well as to the xCPS system in Reference [11]. Further, we formalized a non-essential requirement of the wafer handler as a p-attracting constraint automaton and showed that its addition to the specification results in the reduction of the optimization-space of $60 \%$ compared with the original state-space and for which the optimal result was preserved.

There are a number of research directions to extend this work. An interesting direction would be to perform state-space and optimization-space expansion in tandem with makespan optimization. Such an on-the-fly approach would avoid the explicit construction of the state-space and optimization-space of a logistics automaton. Furthermore, partial-order reduction techniques, such as the one proposed in Reference [20], could be applied to avoid the exploration of redundant sequences. Another interesting research direction would be the generalization of this algebra and its application to finite-state automata. Moreover, the specification of latency requirements has not been explored in the work presented, which constitutes an important research direction to allow a broader class of flexible manufacturing systems to be specified. Finally, more case studies (e.g., in the printing domain) would help to investigate the limitations of the approach and to generalize it.

\section{REFERENCES}

[1] Marianne Akian, Ravindra Bapat, and Stephane Gaubert. 2006. Max-plus algebra. Disc. Math. Applic. 39 (11 2006). DOI : https://doi.org/10.1201/9781420010572.ch25.

[2] Chaudhry Imran Ali and Khan Abid Ali. 2016. A research survey: Review of flexible job shop scheduling techniques. Int. Trans. Op. Res. 23, 3 (2016), 551-591.

[3] Ali Allahverdi. 2016. A survey of scheduling problems with no-wait in process. Eur. f. Op. Res. 255, 3 (2016), 665-686.

[4] Ali Allahverdi, C. T. Ng, T. C. E. Cheng, and Mikhail Y. Kovalyov. 2008. A survey of scheduling problems with setup times or costs. Eur. F. Op. Res. 187, 3 (2008), 985-1032.

[5] Francois Baccelli, Guy Cohen, Geert Jan Olsder, and Jean-Pierre Quadrat. 2001. Synchronization and linearity: An algebra for discrete event systems.

[6] J. Bastos, J. P. M. Voeten, S. Stuijk, H. Corporaal, and R. R. H. Schiffelers. 2018. Exploiting specification modularity to prune the optimization-space of manufacturing systems. In Proceedings of the Software and Compilers for Embedded Systems Conference (SCOPES'18).

[7] Kris Braekers, Katrien Ramaekers, and Inneke Van Nieuwenhuyse. 2016. The vehicle routing problem: State of the art classification and review. Comput. Industr. Eng. 99 (2016), 300-313.

[8] Ed Brinksma. 1990. Constraint-oriented specification in a constructive formal description technique. In Stepwise Refinement of Distributed Systems Models, Formalisms, Correctness, J. W. de Bakker, W.-P. de Roever, and G. Rozenberg (Eds.). Springer Berlin Heidelberg, 130-152. https://link.springer.com/chapter/10.1007\%2F3-540-52559-9_63.

[9] R. de Groote, J. Kuper, H. Broersma, and G. J. M. Smit. 2012. Max-plus algebraic throughput analysis of synchronous dataflow graphs. In Proceedings of the 38th Euromicro Conference on Software Engineering and Advanced Applications. $29-38$.

[10] Robert Doering and Yoshio Nishi. 2007. Handbook of Semiconductor Manufacturing Technology. Vol. 32. CRC Press.

[11] Twan Basten et al. 2020. Scenarios in the Design of Flexible Manufacturing Systems. Springer International Publishing, Cham, 181-224. DOI : https://doi.org/10.1007/978-3-030-20343-6_9.

[12] S. T. J. Forschelen, J. M. van de Mortel-Fronczak, Rong Su, and J. E. Rooda. 2012. Application of supervisory control theory to theme park vehicles. Disc. Event Dynam. Syst. 22, 4 (2012), 511-540.

[13] Bernd Heidergott, Geert Jan Olsder, and Jacob van der Woude. 2006. Max Plus at Work: Modeling and Analysis of Synchronized Systems: A Course on Max-Plus Algebra and Its Applications. Princeton University Press.

[14] Robin Milner. 1983. Calculi for synchrony and asynchrony. Theoret. Comput. Sci. 25, 3 (1983), 267-310.

[15] J. P. Nogueira Bastos. 2018. Modular Specification and Design Exploration for Flexible Manufacturing Systems. Ph.D. Dissertation. Technical University of Eindhoven, The Netherlands.

[16] Peter J. Ramadge and W. Murray Wonham. 1987. Supervisory control of a class of discrete event processes. SIAM 7 . Contr. Optim. 25, 1 (1987), 206-230.

[17] Ofira Shmueli, Nava Pliskin, and Lior Fink. 2015. Explaining over-requirement in software development projects: An experimental investigation of behavioral effects. Int. f. Project Manag. 33, 2 (2015), 380-394. 
[18] Dirk A. van Beek, Wan Fokkink, D. Hendriks, A. Hofkamp, Jasen Markovski, J. M. van de Mortel-Fronczak, and Michel A. Reniers. 2014. CIF 3: Model-based engineering of supervisory controllers. In Proceedings of the International Conference on Tools and Algorithms for the Construction and Analysis of Systems.

[19] B. van der Sanden, J. Bastos, J. Voeten, M. Geilen, M. Reniers, T. Basten, J. Jacobs, and R. Schiffelers. 2016. Compositional specification of functionality and timing of manufacturing systems. In Proceedings of the Forum on Specification and Design Languages.

[20] Bram van der Sanden, Marc Geilen, Michel A. Reniers, and Twan Basten. 2018. Partial-order reduction for performance analysis of max-plus timed systems. In Proceedings of the 18th International Conference on Application of Concurrency to System Design.

[21] J. van Pinxten, M. Geilen, T. Basten, U. Waqas, and L. Somers. 2016. Online heuristic for the multi-objective generalized traveling salesman problem. In Proceedings of the Design, Automation Test in Europe Conference Exhibition (DATE'16). 822-825.

[22] Joost van Pinxten, Umar Waqas, Marc Geilen, Twan Basten, and Lou Somers. 2017. Online scheduling of 2-re-entrant flexible manufacturing systems. ACM Trans. Embed. Comput. Syst. 16, 5s (Sept. 2017).

[23] U. Waqas. 2017. Scheduling and Variation-aware Design of Self-re-entrant Flowshops. Ph.D. Dissertation. Technische Universiteit Eindhoven.

Received May 2019; revised June 2020; accepted September 2020 